Article

\title{
Transient Differentiation Maximum Power Point Tracker (Td-MPPT) for Optimized Tracking under Very Fast-Changing Irradiance: A Theoretical Approach for Mobile PV Applications
}

\author{
Roberto I. Rico-Camacho ${ }^{1}$, Luis J. Ricalde ${ }^{1, *}$, Ali Bassam ${ }^{1}{ }^{\mathbb{D}}$, Manuel I. Flota-Bañuelos ${ }^{1}$ and Alma Y. Alanis ${ }^{2} \mathbb{D}$ \\ 1 Faculty of Engineering, Universidad Autonoma de Yucatan, Av. Industrias No Contaminantes, \\ Merida 97110, Mexico; a14015458@alumnos.uady.mx (R.I.R.-C.); baali@correo.uady.mx (A.B.); \\ manuel.flota@correo.uady.mx (M.I.F.-B.) \\ 2 Centro Universitario de Ciencias Exactas e Ingenierias (CUCEI), Universidad de Guadalajara, \\ Guadalajara 44430, Mexico; almayalanis@gmail.com \\ * Correspondence: 1ricalde@correo.uady.mx
}

Citation: Rico-Camacho, R.I.; Ricalde L.J.; Bassam, A.; Flota-Bañuelos, M.I.; Alanis, A.Y. Transient Differentiation Maximum Power Point Tracker (Td-MPPT) for Optimized Tracking under Very Fast-Changing Irradiance: A Theoretical Approach for Mobile PV Applications. Appl. Sci. 2022, 12, 2671. https://doi.org/10.3390/ app12052671

Academic Editor: Isabel Santiago Chiquero

Received: 31 December 2021

Accepted: 25 February 2022

Published: 4 March 2022

Publisher's Note: MDPI stays neutral with regard to jurisdictional claims in published maps and institutional affiliations.

Copyright: () 2022 by the authors Licensee MDPI, Basel, Switzerland. This article is an open access article distributed under the terms and conditions of the Creative Commons Attribution (CC BY) license (https:// creativecommons.org/licenses/by/ $4.0 /)$.

\begin{abstract}
This work presents an algorithm for Maximum Power Point Tracking (MPPT) that measures transitory states to prevent drift issues and that can reduce steady-state oscillations. The traditional MPPT algorithms can become confused under very fast-changing irradiance and perform tracking in the wrong direction. Errors occur because these algorithms operate under the assumption that power changes in the system are triggered exclusively due to perturbations introduced by them. However, the power increase triggered by irradiance changes could be more significant than those caused by the perturbation effect. The proposed method modifies the Perturb and Observe algorithm $(\mathrm{P} \& \mathrm{O})$ with an additional measurement stage performed close to the maximum overshoot peak after the perturbation stage. By comparing power changes between three measurement points, the algorithm can accurately identify whether the perturbation was made in the correct direction or not. Furthermore, the algorithm can use additional information to determine if the operating point after the perturbation stage is beyond the maximum power point (MPP) and perturb in the opposite direction for the next iteration. Thus, the proposed algorithm shows reduced steady-state oscillations and improved tracking under fast irradiance changes compared to conventional $\mathrm{P} \& \mathrm{O}$ and $\mathrm{P} \& \mathrm{O}$ with power differences ( $\mathrm{dP}-\mathrm{P} \& \mathrm{O})$. The design is validated via simulations using fast-changing irradiance tests based on the standard EN 50530 accelerated by a factor of $100 \times$. The proposed algorithm achieved $99.74 \%$ of global efficiency versus $97.4 \%$ of the classical $\mathrm{P} \& \mathrm{O}$ and $99.54 \%$ of the $\mathrm{dP}-\mathrm{P} \& \mathrm{O}$ under the tested conditions.
\end{abstract}

Keywords: MPPT; photovoltaic system; perturb and observe; transient differentiation; fast irradiance

\section{Introduction}

Solar Photovoltaic (PV) panels operation can be described with a characteristic PVcurve, which clearly shows the Maximum Power Point (MPP). The panels should operate as close to this point as possible to obtain the maximum power available even in the presence of MPP variation due to external factors such as irradiance and temperature. Thus, Maximum Power Point Tracking (MPPT) systems are implemented with algorithms to automatically locate the desired operating voltage or current that produces the maximum power available. Several MPPT techniques, such as curve-fitting, fractional short-circuit current, look-up table, differentiation technique, perturbation and observation ( $\mathrm{P} \& \mathrm{O})$, and variable-weather-parameter, among others, are present in the literature [1-4]. Two of the most employed in practice and commonly reported MPPT algorithms are $\mathrm{P} \& \mathrm{O}$ and incremental conductance (IncCond). Furthermore, a detailed analysis in [5] showed that IncCond could be treated as a specific implementation of $\mathrm{P} \& \mathrm{O}$. 
P\&O has been widely employed due to its relatively simple structure, reasonably good performance, easy implementation on low-cost digital controllers, and no dependence on previous information about the system or environmental measurements [3]. Nonetheless, this algorithm suffers from two main drawbacks, as pointed out in [6,7]. First, the operating point oscillates around MPP, reducing overall efficiency, as it needs to keep perturbing a fixed increment $(\delta)$ to track MPP. Second, it can becomes confused and tracks in the wrong direction during fast irradiance changes in an issue commonly referred to in the MPPT literature as drift [8]. A modification of $\mathrm{P} \& \mathrm{O}$ that addresses these two issues while maintaining its advantages could improve the efficiency of current systems without requiring significant hardware changes.

To reduce the issue of oscillations around MPP during steady irradiance, dynamic perturbation sizes have been proposed [9-13]. These algorithms use the ratio between power change observed and perturbation size $(\Delta \mathrm{p} / \delta)$ to establish the next perturbation size. Since the PV curve abruptly decreases around MPP, the perturbations introduced by MPPT are reduced as the system moves closer to MPP. However, this solution might exhibit some disadvantages under rapidly varying irradiance conditions, as significant variations on perturbation size may occur. In [14], constraining the increment size to a reasonable range by introducing a logarithmic function is proposed. This modification keeps the perturbation size small even if the ratio, $\Delta \mathrm{p} / \delta$, grows. Nevertheless, even if the oscillation is reduced, the loss of tracking direction is not addressed.

Several modifications to the $\mathrm{P} \& \mathrm{O}$ algorithm have been proposed to solve the drift issue [15-21]. The use of boundary conditions combined with dynamic perturbation sizes has been suggested to prevent the system from drifting away too far from the MPP by $[15,16]$. Although this reduces efficiency losses, the system still drifts in between the MPP and the boundary condition. In [17], a method to detect irradiance changes using fractional short circuit current (FSCC) while reducing the perturbation size is presented. In [18], a modified $\mathrm{P} \& \mathrm{O}$ for drift avoidance adds a current condition to the traditional $\mathrm{P} \& \mathrm{O}$ when it is more prone to fail. The proposed algorithm consists of three stages. First, the short circuit current is measured, and MPP is calculated as a fraction of it. Second, a regular $\mathrm{P} \& \mathrm{O}$ searches the MPP starting at the operating point estimated in the first stage. In the final stage, at each iteration of $\mathrm{P} \& \mathrm{O}$, the module current is compared with FSCC. If a change over a certain threshold is detected, the system returns to the first stage. The use of a smaller perturbation size reduced steady-state oscillations. The algorithm showed better tracking during irradiance changes than traditional P\&O. Although $[17,18]$ reduced steady-state oscillations, an inherent loss of power occurs in the operation of FSCC. Adding extra samples associated with a combination of perturbations has been proposed in [19] to isolate the effects in power caused by irradiance (or temperature) fluctuations from the overall power change observed after MPPT perturbation. MPPT then considers this additional information to make a better decision on the next perturbation direction. This method showed better efficiencies during rapid irradiance changes than $\mathrm{P} \& \mathrm{O}(98.78 \%$ compared to $93.31 \%$ ). However, the additional perturbation stage effectively divides $T_{M P P T}$. The division occurs because several perturbations need to be made before making a control decision. In $[20,21]$, the authors propose an algorithm based on the traditional P\&O (dp$\mathrm{P} \& \mathrm{O})$ with an additional power measurement stage in the middle of each perturbation period $\left(T_{M Р P T} / 2\right)$ to calculate the direction in irradiance changes. Since MPPT does not perform any action between perturbation periods, it presumes that any observed power change is exclusively due to external factors. Although, experimental data showed that the algorithm would still suffer some degree of drifting from MPP.

Traditional MPPT algorithms either wait for the system to reach a steady-state before measuring power changes or compute an average of numerous measurements during $T_{M P P T}$ to remove ripple components. Nonetheless, methods that consider natural oscillations occurring in the converters show good dynamic tracking [5,22-24]. It has been observed that the continuous oscillations around the MPPT produced by these schemes 
can decrease steady-state efficiency. Furthermore, the added complexity makes them more challenging to implement than compared with traditional MPPTs.

This study presents an improvement to the classical P\&O that prevents MPPT from drifting during fast irradiance changes and steady-state oscillations around the MPP by calculating power derivatives during transitory states. Using an additional measurement in the PV curve, the proposed algorithm computes the derivative between the previous operating point and the maximum overshoot peak $\left(d p_{1}\right)$ and between the maximum overshoot and the final operating point $\left(d p_{2}\right)$. Because of this, the proposed method is referred to as transient differentiation MPPT (Td-MPPT). When the PV system has underdamped behavior, this algorithm only needs one perturbation around the MPP in each direction instead of the two required by traditional methods. Because of these enhancements, the simulation results showed that efficiency increased compared to the classical $\mathrm{P} \& \mathrm{O}$ and dP-P\&O. Furthermore, the performance is compared to the algorithm in [21] to better illustrate differences between these two approaches. Even though this method addresses tracking a local MPPT such as $\mathrm{P} \& \mathrm{O}$, it can be combined with different techniques such as the fractional short circuit (FSC) [17], the genetic algorithm (GA) [25], artificial bee colony (ABC) [26], particle swarm optimization (PSO) [27], grey wolf optimization (GWO) [28], and enhanced leader particle swarm optimization (ELPSO) [29] to operate under partial shading conditions.

This work is structured as follows: Section two presents classic $\mathrm{P} \& \mathrm{O}$ and dp-P\&O operations. In section three, the reasoning behind the Td-MPPT algorithm and how it calculates the direction of the next perturbation is explained in detail. Then, simulation results of the proposed algorithm and comparison with the method presented in [21] and the traditional $\mathrm{P} \& \mathrm{O}$ are evaluated to demonstrate the added value of the proposed approach. A performance comparison for the three algorithms is carried out for global efficiency during the tests and different irradiance rates of change. Efficiency is calculated according to the definition proposed in the EN50530:2010 standard [30]. Lastly, the conclusions are presented.

\section{Classical $P \& O$ and $d p-P \& O$}

The classical $\mathrm{P} \& \mathrm{O}$ algorithm is presented in Figure 1. This method has been widely employed due to its simplicity, relatively good performance, and generic implementation. The actions are executed for every discrete k-sampling period (usually at a given fixed interval $T_{M P P T}$ ) to detect the PV-curve slope and determine the MPP's location. In what follows, subindex $\mathrm{k}$ points out the specific value for each iteration period, e.g., $v_{k}$ and $p_{k}$ are the voltage and power of the PV module at the $\mathrm{k}_{\text {-th }}$ iteration. $\mathrm{P} \& \mathrm{O}$ detects the slope by measuring the power difference $\left(\Delta p=p_{k}-p_{k-1}\right)$ after adding or subtracting a fixed size perturbation $(\delta)$ to the operating point (usually in voltage). If $\Delta p$ is positive, it is deduced that the perturbation moved the operating point closer to the MPP (uphill move) and that $\delta$ has to have the same sign for the next one, i.e., $\Delta p / \delta>0$ on the left side of the MPP and $\Delta p / \delta<0$ on the right side of the MPP. 


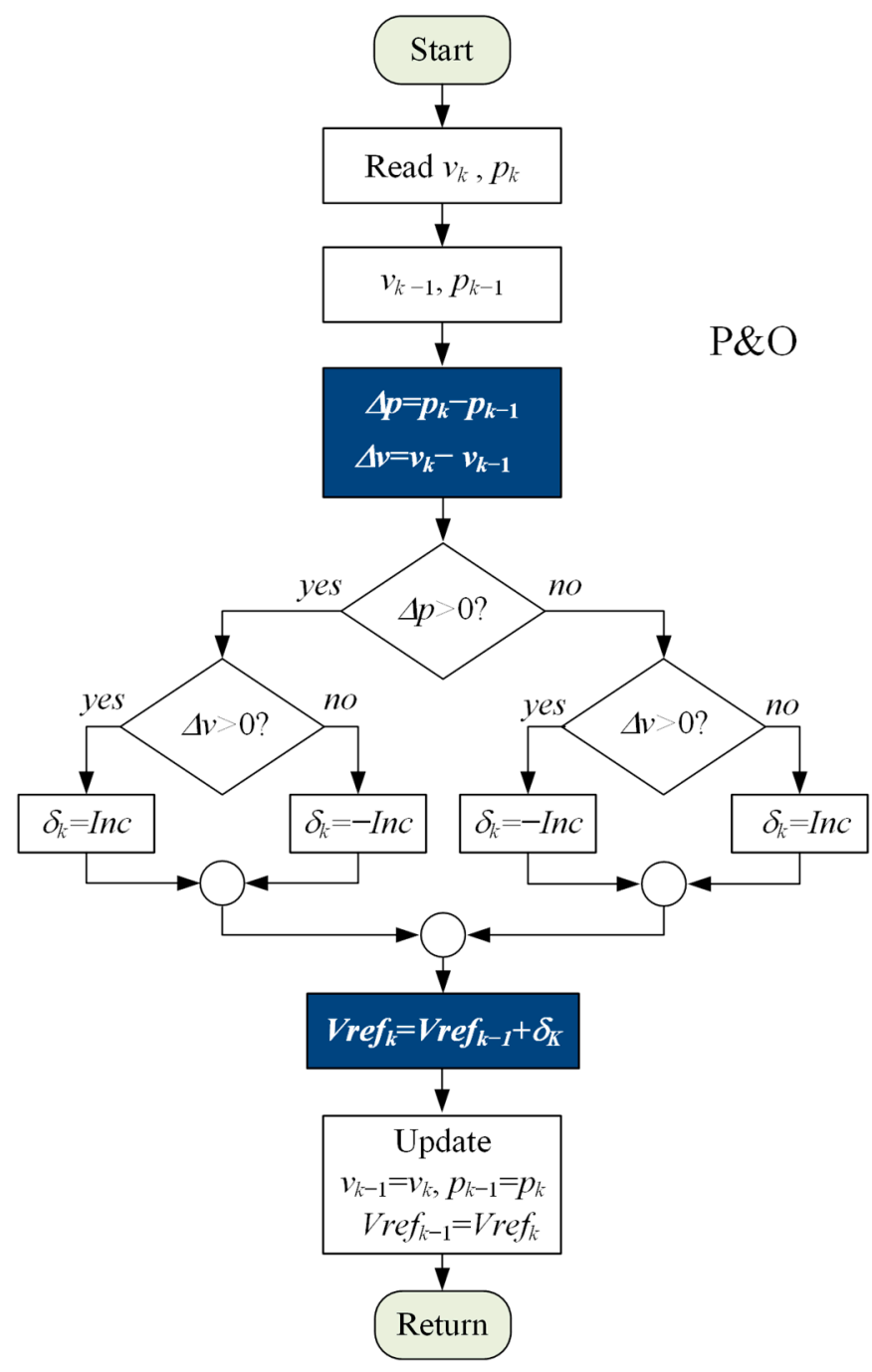

Figure 1. Flowchart of the classical P\&O MPPT.

\subsection{Issues under Rapidly Changing Irradiance}

As previously mentioned, it has been reported that the $\mathrm{P} \& \mathrm{O}$ method can become confused under rapidly changing irradiance scenarios $[5,16,19,31]$. This issue occurs because the algoritm assumes that the change observed in power output is triggered exclusively by the perturbation of MPPT. Nevertheless, if a significant enough shift in irradiance occurs, it can have an even more substantial effect on power variation than MPPT-induced perturbation. Therefore, the algorithm drifts away from the MPPs reported in $[8,18,19]$. The classical drift behavior is illustrated in Figure 2. A steady irradiance increment over time is shown, the MPP line (red dot) ramps up, and the system power (solid) increases along with it. At the starting operation point $p_{k-2}$, increment $\delta$ in the wrong direction is introduced by the MPPT driving the power away from the MPP line. During the next perturbation stage, it can be observed that power $p_{k-1}$ is greater than the previous power state $p_{k-2}$ due to the rapidly increasing irradiance. Since $\Delta p_{-1}>0$, the classical $\mathrm{P} \& \mathrm{O}$ algorithm decides to apply an increment in the wrong direction for the second time. Notice that since $\Delta p>0$, the P\&O method would keep driving the operating point away from MPP for future iterations. This would be repeated until the effect caused by the controller action is greater than the impact of external factors. An approach to determine whether the perturbation was made in the right direction is presented in this work. 


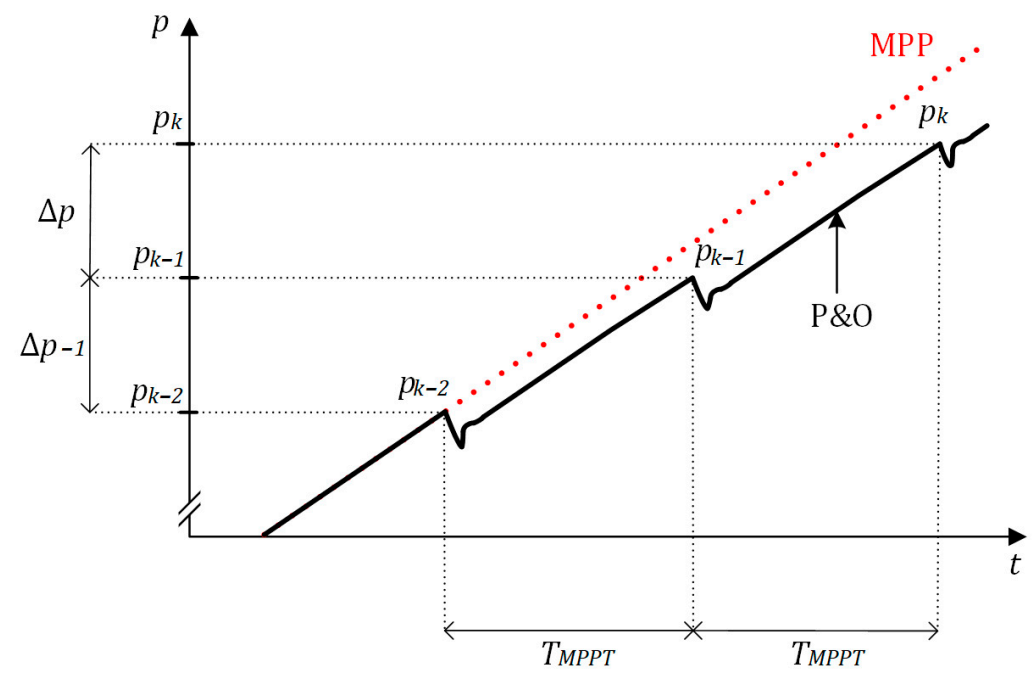

Figure 2. Classical drifting behavior of $\mathrm{P} \& \mathrm{O}$ around MPP under increasing irradiance, as reported in the literature.

\section{2. $d p-P \mathcal{E O}$ Modification}

The dP-P\&O method performs an additional power measurement $\left(p_{x}\right)$ in the middle of the perturbation period $\left(T_{M P P T} / 2\right)$. It can be assumed that any power difference $\left(d p_{2}\right)$ between the power before the next perturbation $\left(p_{k}\right)$ and $p_{x}$ is solely due to external causes since the operating point remains the same. In contrast, the power difference $\left(d p_{1}\right)$ between the perturbation point $\left(p_{k-1}\right)$ and $p_{x}$ contains the change caused by the action of the algorithm and the irradiance change. Based on this premise, the power difference obtained from the MPPT operation can be calculated as follows.

$$
d p=d p_{1}-d p_{2}=\left(p_{x}-p_{k-1}\right)-\left(p_{k}-p_{x}\right)=2 p_{x}-p_{k}-p_{k-1} .
$$

However, under rapid irradiance changes, the operating point may fluctuate without the MPPT operation because of parasitic resistances in the converter. This fluctuation could confuse the controller since the premise of the process would not be valid.

\section{Proposed Td-MPPT Method}

The idea behind the proposed method is that MPP can be tracked better if there is more information about the PV-curve. Traditional MPPT algorithms perturb every update interval $\left(T_{M P P T}\right)$ and either wait for the system to reach a steady-state or filter natural oscillations before registering power changes. Nevertheless, algorithms can enrich the information they rely on by sampling transitory states. However, implementation complexity increases if too many points are registered. By selecting key measurement points, enough data to provide an effective MPP tracking can be gathered while preserving a relatively simple algorithm.

The method presented in this investigation features an additional measurement stage during the transient state to the conventional $\mathrm{P} \& \mathrm{O}\left(p_{x}\right)$. The added measurement is registered during the overshoot produced after the perturbation made by MPPT, as observed in (Figure 3). This improvement prevents the drift and oscillation characteristic of the P\&O algorithm while maintaining its relative simplicity. 


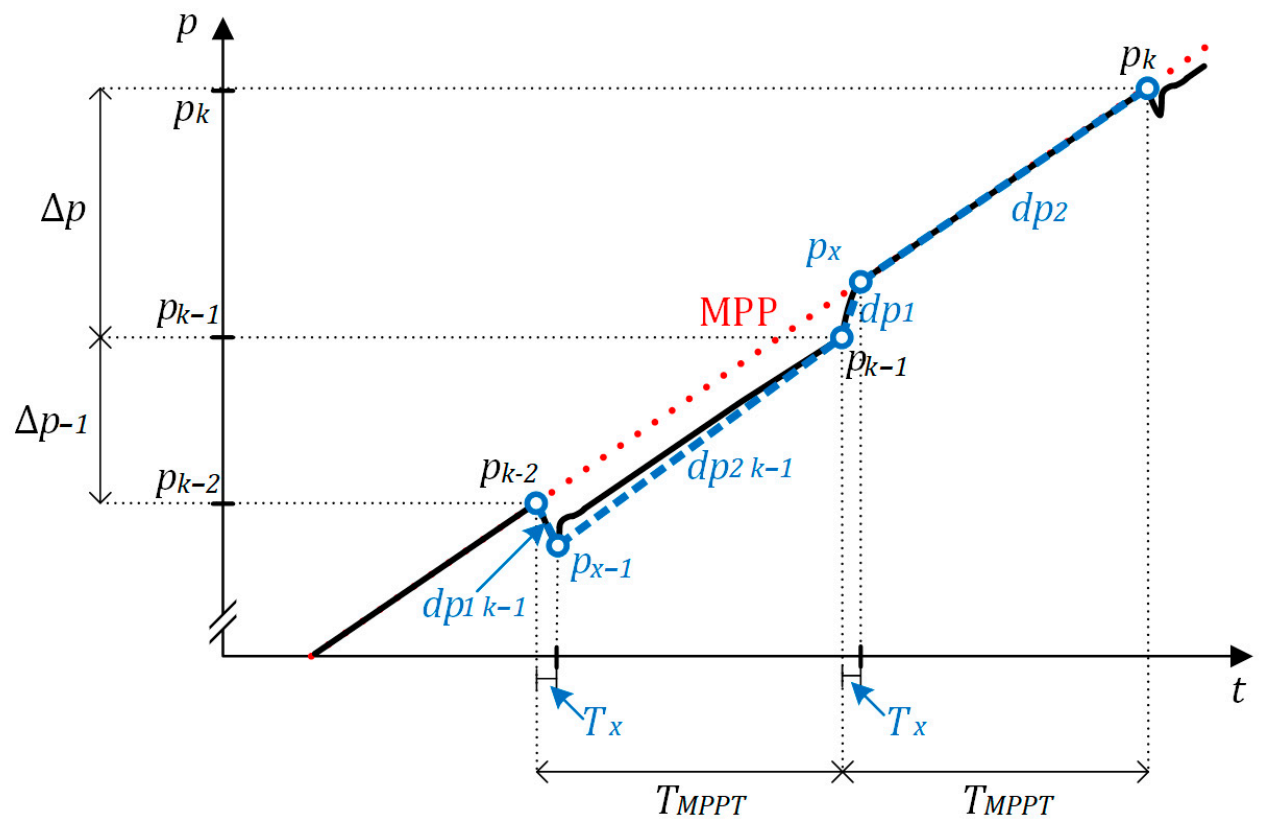

Figure 3. Perturbation performed by the Td-MPPT algorithm around MPP under increasing irradiance.

Figure 3 shows the proposed algorithm influence on power and voltage under the steadily increasing irradiance scenario previously shown in Figure 2. By registering three different operation points, the proposed algorithm calculates two differentials of power for each perturbation period as follows.

$$
\begin{gathered}
d p_{1}=\frac{p_{x}-p_{k-1}}{T_{x}} \\
d p_{2}=\frac{p_{k}-p_{x}}{T_{M P P T}-T_{x}}
\end{gathered}
$$

During the first perturbation period, $d p_{1 k-1}$ and $d p_{2 k-1}$ are calculated. It can be observed that $d p_{1 k-1}$ is drastically reduced when the perturbation introduced by the algorithm drives the operating point away from the MPP even under rapidly changing irradiance scenarios. In contrast, $d p_{2 k-1}$ depends much more on the effects of irradiance changes. For this case, it can be stated that $d p_{2 k-1}>d p_{1 k-1}$ when the perturbation moved the system away from the MPP. Then, Td-MPPT executes the next perturbation in the opposite direction. In contrast, the perturbation performed at $p_{k-1}$ brings the power closer to MPP and the following is the case:

$$
d p_{1}>d p_{2}
$$

when the perturbation is performed in the appropriate direction, assuming that the change rate in irradiance remains constant during a sample period. To reduce computational burden, $T_{M P P T}$ can be selected as an integer multiple of the overshoot measuring time $T_{x}$; thus, condition (3) can be expressed as follows.

$$
\begin{gathered}
\left(p_{x}-p_{k-1}\right) \cdot(\alpha-1)>p_{k}-p_{x} . \\
\alpha=\frac{T_{M P P T}}{T_{X}}
\end{gathered}
$$

Figure 4 shows the classical, usually underdamped, transient response of a direct control MPPT [1-4] oscillating around the MPP during steady irradiance conditions. The system operating at a point on the right of the MPP $\left(v_{k-1}\right)$ is perturbed in the correct direction by the MPPT to a point on the left of it $\left(v_{k}\right)$. The classical P\&O method uses only 
$\Delta p$ to decide whether the perturbation was made in the correct direction or not. For this example, since $\Delta p>0, \mathrm{P} \& \mathrm{O}$ would assume that the next perturbation should be made in the same direction. However, doing so would drive the operating point further away from MPP since $v_{k}$ is already to the left of MPP. This phenomenon is why $\mathrm{P} \& \mathrm{O}$ is known to oscillate between three operating points around MPP. It can be noted that the overshoot can provide additional information about the PV-curve shape since it occurs in the same direction as $\delta_{\mathrm{k}}$. By applying this principle, Td-MPPT can decide whether perturbing in the same direction again would bring the system closer to MPP or not. Similarly to the rapidly changing irradiance scenario, it can be assumed that the perturbation was made in the correct direction if $d p_{1}>d p_{2}$ under steady irradiance, i.e., MPP is beyond $v_{k}$ and MPPT should keep perturbating in the same direction to reach it. On the contrary, if $d p_{1}<d p_{2}$, then the proposed MPPT can assume that $v_{k}$ is closer to MPP than $v_{x}$ so the next perturbation needs to be performed in the opposite direction. Td-MPPT would then reduce steady-state oscillations by alternating between two operating points around the MPP instead of three.

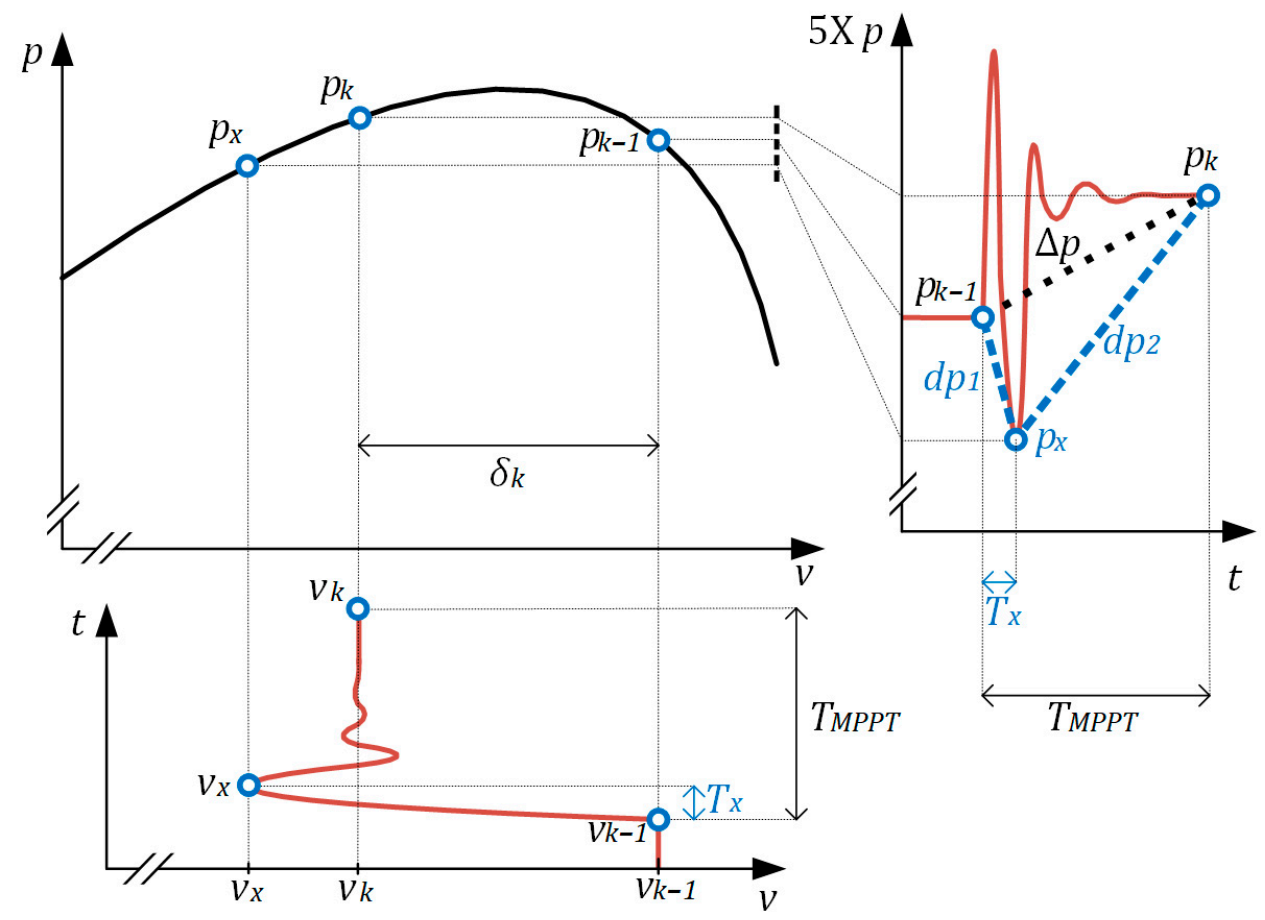

Figure 4. PV system voltage and power transient response after perturbation around the MPP under steady irradiance.

The flowchart of the overall Td-MPPT is shown in Figure 5. It becomes evident that $T_{x}$ and $T_{M P P T}$ need to be determined to tune the Td-MPPT algorithm properly. To simplify the required computations, $T_{M P P T}$ should be selected as a multiple of $T_{x}$. Traditional MPPT algorithms might present erratic behavior caused by transient states if they perturb the operating point too quickly. A safe approach would be to use a $T_{M P P T}$ that is considerably larger than the natural oscillation period of the system. For a DC-DC boost converter (4) with capacitance $(C)$ and inductance $(L), T_{M P P T}$ can be selected to satisfy the following.

$$
T_{M P P T} \gg 2 \pi \sqrt{L C} \text {. }
$$

However, if the perturbation time is too slow, the MPPT cannot keep up with fast irradiation changes. The authors in [32-34] have carried out an analysis on the dynamic behavior of PV systems and their converters to optimize $T_{M P P T}$ and improve the dynamic response of traditional MPPT systems. In addition to optimizing $T_{M P P T}$ for the proposed algorithm, an analysis of the system's transient response would allow choosing the op- 
timum time to observe the maximum overshoot caused by the step perturbation. The research in [31] showed that the transient behavior of PV systems changes depending on the operating region of the system, i.e., constant current region (CCR), constant power region (CPR), and constant voltage region (CVR).

When analyzed as a small signal equivalent circuit, the simulated converter (Figure 6) contains two independent inputs: the control input $(\hat{d})$ and the load current $(\hat{i})$. The variables with hats represent the small-signal changes around steady-state values. Hence, for an ideal boost converter, the voltage output $(\hat{v})$ can be expressed as a superposition of these to find the following: the small control signal to array voltage transfer function $\left(G_{v}\right)$ and the load current to array voltage output transfer function $\left(G_{V f}\right)$ as follows:

$$
\hat{v}=G_{v} \hat{d}+G_{v f} \hat{i}
$$

where the following is the case.

$$
\begin{gathered}
G_{v}=\frac{V_{f}}{L C s^{2}+\left(\frac{L}{R(1-D)^{2}}\right) s+1} \\
G_{V f}=\frac{R(1-D)}{L C R C_{O} S^{3}+\left(C+\frac{C_{O}}{(1-D)^{2}}\right) L s^{2}+\left(R\left(C(1-D)^{2}+C_{O}\right)+\frac{L}{R(1-D)^{2}}\right) s+2}
\end{gathered}
$$

Small duty-cycle changes can be expressed as follows.

$$
\hat{d}=\frac{\Delta D}{s}
$$

For this application (Figure 6), the steady-state value of the duty cycle $(D)$ is determined by the relationship between the voltage reference defined by the MPPT algorithm $\left(V_{r e f}\right)$, and the output voltage of the power supply $\left(V_{f}\right)$ as follows

$$
D=1-\frac{V_{r e f}}{V_{f}}
$$

Transfer function $G_{V f}$ is relevant for applications such as grid-tied systems since it models interfacing converter effects on dynamic behavior [33,34]. However, for an application with no variation in the load current, transfer function $G_{V f}$ is equal to 0 .

For an ideal boost converter, $R_{i n}=R(1-D)^{2}$; thus, $G_{v}$ assumes the following:

$$
G_{v}=\frac{\mu \omega_{n}^{2}}{s^{2}+2 \xi \omega_{n} s+\omega_{n}^{2}}
$$

where $\mu=-V_{O}, \omega_{n} \approx 1 / \sqrt{L C}$ and $\xi \approx \sqrt{L C} /\left(2 R_{\text {in }}\right)$.

It must be noted that $R_{\text {in }}$ denotes the ratio between the PV panel voltage and current, and it depends on each panel's characteristics, the operating point (CCR, CPR, or CVR), and external conditions such as temperature and irradiance [31]. Because of this, the maximum overshooting time is not fixed, and under certain circumstances, a direct control MPPT cannot not show underdamped behavior. It must be noted that the Td-MPPT algorithm would avoid drift even under these conditions because of the principle behind algorithms that perform additional measurements as $[19,20]$. Furthermore, it would operate as a regular $\mathrm{P} \& \mathrm{O}$ under steady-state irradiance, performing two perturbations in the same direction around the MPP since the additional measurement would not provide additional information about the PV-curve. In this research, the natural oscillation frequency of the DC-DC converter will be used to approximate $T_{x}$. An autotune stage can be added at system startup to detect $T_{x}$ for a specific system. 


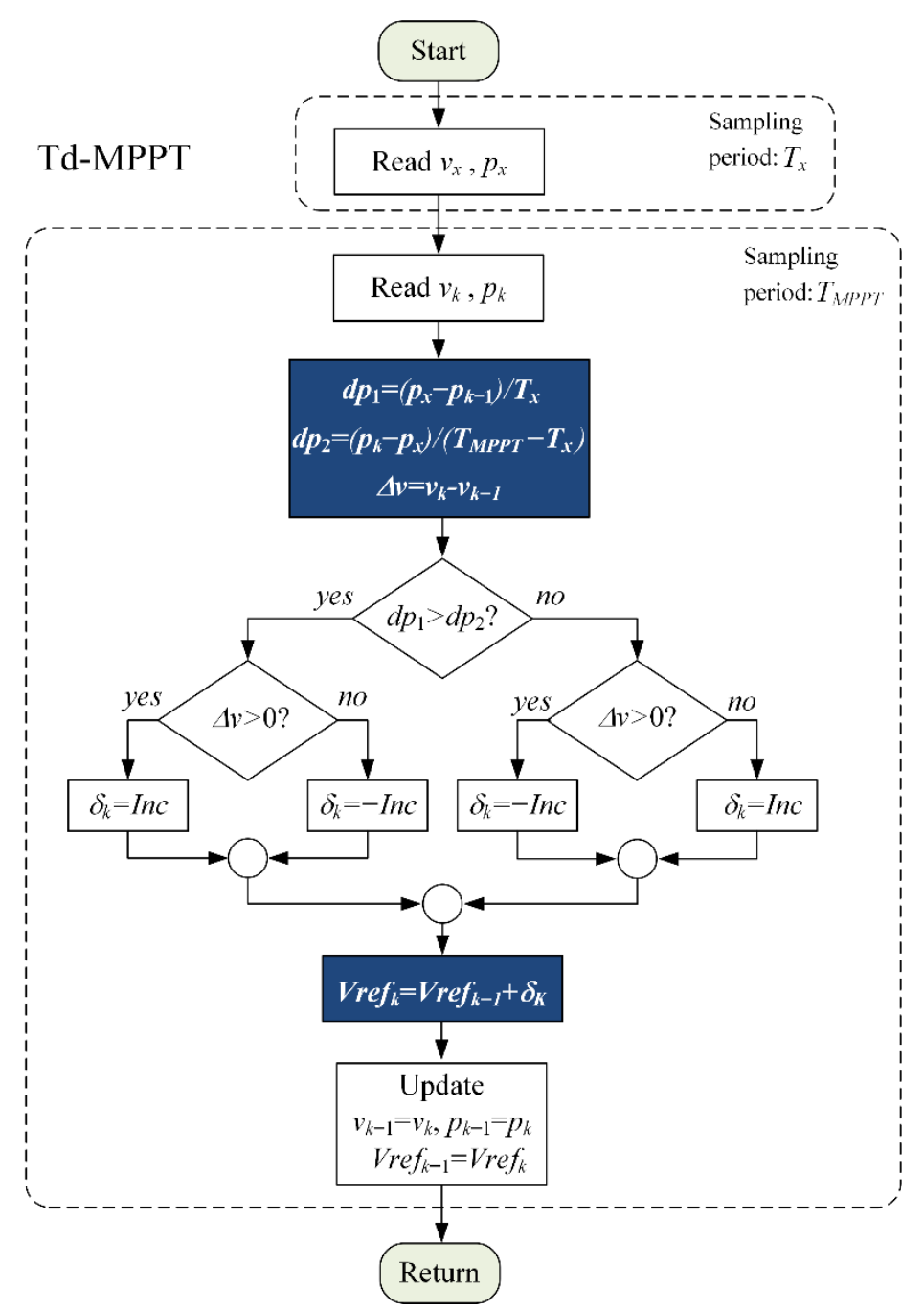

Figure 5. Flowchart of the proposed Td-MPPT algorithm.

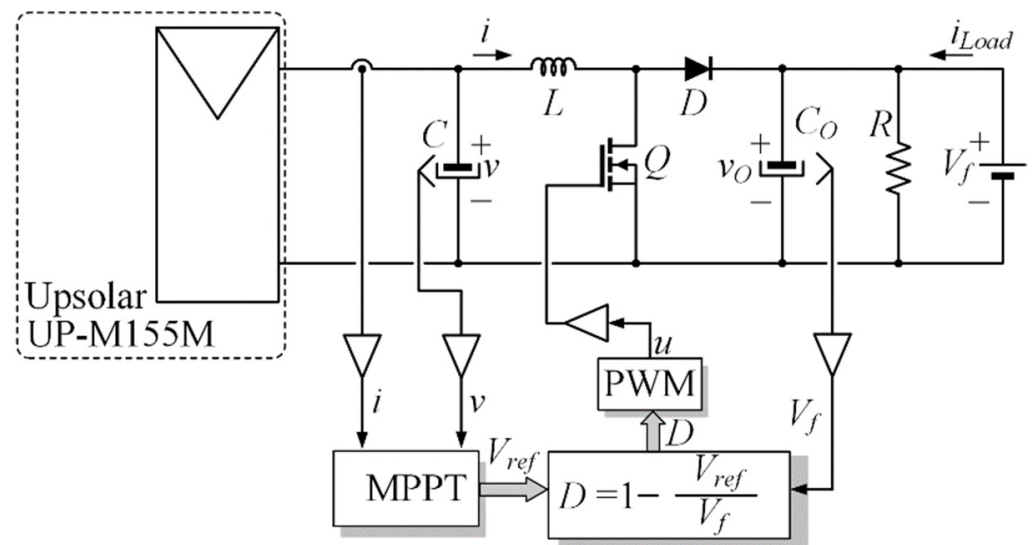

Figure 6. Test platform for simulations.

The proposed algorithm was designed to not require additional sensors compared to the traditional $\mathrm{P} \& \mathrm{O}$. The added computational burden remains marginal as it only requires two extra multiplications compared to the conventional $\mathrm{P} \& \mathrm{O}$ algorithm if the condition specified in equation 4 substitutes the division to calculate $d p_{1}$ and $d p_{2}$. As specified in [34], P\&O can be implemented in a low-cost embedded board such as an STM32F103 Blue pill board. A system based on this low-cost microcontroller requires only one clock cycle per 
multiplication [34], meaning that would only need two additional clock cycles compared to the implementation of $\mathrm{P} \& \mathrm{O}$. For this reason, a system designed to operate using the classical P\&O could be reprogramed to run Td-MPPT at no extra cost.

Selecting a microcontroller with at least two hardware analog to digital converters (ADC) is crucial for obtaining the maximum tracking speed as it would allow converting two analog signals simultaneously. The three algorithms in this work would benefit from this feature as they use the same two sensors to operate. However, this is even more important for the Td-MPPT as it samples a rapid change transient response.

Since the Td-MPPT algorithm is based on a conventional approach, it is unable to guarantee tracking of the global maximum power point (GMPP) under nonuniform irradiance. A hybrid algorithm between a GMPP search technique and the proposed algorithm could be implemented to solve this shortcoming. These hybrid algorithms have shown promising results in tracking GMPP by using conventional hill-climbing MPPT hybrid algorithms [17,25-29]. Nonetheless, these techniques involve a higher computational burden and would require a more powerful microcontroller due to the high number of divisions, multiplication, summation of series of numbers, and power calculations used in their structure [34]. However, some are more affordable techniques than others [35]; thus, a combination of a relatively inexpensive GMPP approach and Td-MPPT could be implemented at a lower cost than algorithms with equivalent efficiency.

\section{Simulation Results and Discussion}

The proposed Td-MPPT algorithm is simulated in MATLAB/Simulink to compare it with the traditional $\mathrm{P} \& \mathrm{O}$ and $\mathrm{dp}-\mathrm{P} \& \mathrm{O}$ to contrast their performance and illustrate the differences between them. The algorithms are simulated using irradiance profiles based on the ones defined in the efficiency test of the EN50530 standard [30] to compare their performance under different scenarios (Table 1).

Table 1. Irradiance profile as described in the EN50530 standard.

\begin{tabular}{|c|c|c|c|c|c|c|c|}
\hline \multicolumn{8}{|c|}{ a. Irradiance low to medium $\left(100 \mathrm{~W} / \mathrm{m}^{2}-500 \mathrm{~W} / \mathrm{m}^{2}\right)$} \\
\hline $\begin{array}{c}\text { Settling } \\
{[\mathrm{s}]}\end{array}$ & $\begin{array}{l}\text { Rep. } \\
\text { [n] }\end{array}$ & $\begin{array}{c}\text { Slope } \\
{\left[\mathrm{W} / \mathrm{m}^{2} / \mathrm{s}\right]}\end{array}$ & $\begin{array}{c}\text { Rise } \\
{[\mathrm{s}]}\end{array}$ & $\begin{array}{l}\text { Wait } \\
{[\mathrm{s}]}\end{array}$ & $\begin{array}{c}\text { Fall } \\
{[\mathrm{s}]}\end{array}$ & $\begin{array}{l}\text { Wait } \\
{[\mathrm{s}]}\end{array}$ & $\begin{array}{c}\text { Duration } \\
{[\mathrm{s}]}\end{array}$ \\
\hline 300 & 2 & 0.5 & 800 & 10 & 800 & 10 & 3540 \\
\hline 300 & 2 & 1 & 400 & 10 & 400 & 10 & 1940 \\
\hline 300 & 3 & 2 & 200 & 10 & 200 & 10 & 1560 \\
\hline 300 & 4 & 3 & 133 & 10 & 133 & 10 & 1444 \\
\hline 300 & 6 & 5 & 80 & 10 & 80 & 10 & 1380 \\
\hline 300 & 8 & 7 & 57 & 10 & 57 & 10 & 1372 \\
\hline 300 & 10 & 10 & 40 & 10 & 40 & 10 & 1300 \\
\hline 300 & 10 & 14 & 29 & 10 & 29 & 10 & 1080 \\
\hline 300 & 10 & 20 & 20 & 10 & 20 & 10 & 900 \\
\hline 300 & 10 & 30 & 13 & 10 & 13 & 10 & 760 \\
\hline 300 & 10 & 50 & 8 & 10 & 8 & 10 & 660 \\
\hline & & & & & & Total & 15,936 \\
\hline & & & & & & & 04:25:39 \\
\hline \multicolumn{8}{|c|}{ b. Irradiance medium to high $\left(300 \mathrm{~W} / \mathrm{m}^{2}-1000 \mathrm{~W} / \mathrm{m}^{2}\right)$} \\
\hline $\begin{array}{c}\text { Settling } \\
{[\mathrm{s}]}\end{array}$ & $\begin{array}{l}\text { Rep. } \\
\text { [n] }\end{array}$ & $\begin{array}{c}\text { Slope } \\
{\left[\mathrm{W} / \mathrm{m}^{2} / \mathrm{s}\right]}\end{array}$ & $\begin{array}{c}\text { Rise } \\
{[\mathrm{s}]}\end{array}$ & $\begin{array}{l}\text { Wait } \\
{[\mathrm{s}]}\end{array}$ & $\begin{array}{c}\text { Fall } \\
{[\mathrm{s}]}\end{array}$ & $\begin{array}{c}\text { Wait } \\
{[\mathrm{s}]}\end{array}$ & $\begin{array}{c}\text { Duration } \\
{[\mathrm{s}]}\end{array}$ \\
\hline 300 & 10 & 10 & 70 & 10 & 70 & 10 & 1900 \\
\hline 300 & 10 & 14 & 50 & 10 & 50 & 10 & 1500 \\
\hline 300 & 10 & 20 & 35 & 10 & 35 & 10 & 1200 \\
\hline 300 & 10 & 30 & 23 & 10 & 23 & 10 & 960 \\
\hline 300 & 10 & 50 & 14 & 10 & 14 & 10 & 780 \\
\hline \multirow[t]{3}{*}{300} & 10 & 100 & 7 & 10 & 7 & 10 & 640 \\
\hline & & & & & & Total & 6980 \\
\hline & & & & & & & 01:56:27 \\
\hline
\end{tabular}


As shown in Figure 7, the irradiance profile can be divided into three regions: a low to medium irradiance region (where it fluctuates between $10 \%$ and $50 \%$ of the $\mathrm{G}_{\mathrm{STC}}$ ), a medium to high irradiance region (where it fluctuates between 30\% and 100\%), and a startup region (where it fluctuates between $1 \%$ and $10 \%$ ). Each one comprises a given number of trapezoidal waveforms with varying slopes. The irradiance described in the EN50530 standard is presented in Table 1. For example, during the first trapezoidal waveform, irradiance rises during $800 \mathrm{~s}$ at a rate of $0.5 \mathrm{~W} / \mathrm{m}^{2} / \mathrm{s}$ from $100 \mathrm{~W} / \mathrm{m}^{2}$ to $500 \mathrm{~W} / \mathrm{m}^{2}$, and then it remains steady at $500 \mathrm{~W} / \mathrm{m}^{2}$ during $10 \mathrm{~s}$. Then, it decreases at a rate of $0.5 \mathrm{~W} / \mathrm{m}^{2} / \mathrm{s}$ until it reaches $100 \mathrm{~W} / \mathrm{m}^{2}$. Lastly, it remains steady for another $10 \mathrm{~s}$. The test also includes an additional settling period of $300 \mathrm{~s}$ between each cluster of repetitions with the same slope. The startup region consists of a single trapezoidal profile with an ascending and descending slope of $0.1 \mathrm{~W} / \mathrm{m}^{2} / \mathrm{s}$ with $30 \mathrm{~s}$ settling periods before the first ramp and after each one.

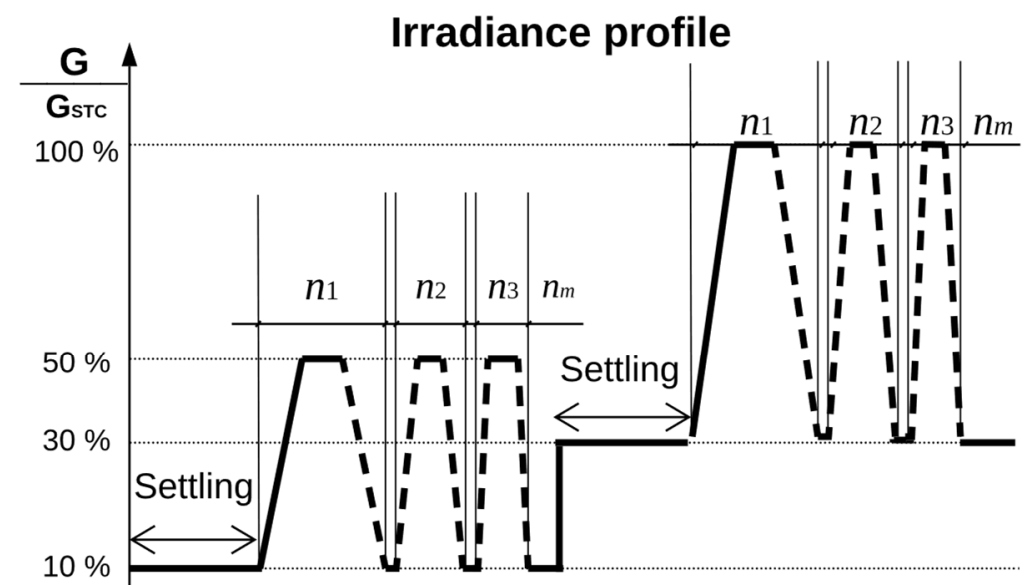

(a) Low to medium irradiance

(b) Medium to high irradiance

Figure 7. Irradiance profile evaluated as described in the EN50530 standard comprising low to medium and medium to high changes as described in Table 1.

To further test the Td-MPPT response under very fast-changing irradiance, a speed multiplier of $10^{2}$ was introduced to the standard EN50530 efficiency test, and the startup test was not included in the simulations. The resulting profiles are shown in Table 2.

The model provided with MATLAB/Simulink of a solar panel UP-M155M made by Upsolar was chosen due to its low series resistance. It includes 72 cells, a temperature coefficient of $\mathrm{V}_{\mathrm{OC}}=-0.40122 \% /{ }^{\circ} \mathrm{C}$, a temperature coefficient of $\mathrm{I}_{\mathrm{SC}}=0.057535 \% /{ }^{\circ} \mathrm{C}$, a light-generated current $\left(\mathrm{I}_{\mathrm{L}}\right)=5.0443 \mathrm{~A}$, a diode saturation current $\left(\mathrm{I}_{0}\right)=6.41 \times 10^{-10}$, a diode ideality factor $=0.98551$, a shunt resistance $\left(R_{\mathrm{SH}}\right)=111.1072 \Omega$, and a series resistance $\left(R_{S}\right)=0.31595 \Omega$. This model has the following electrical characteristics at standard test conditions $\left(\mathrm{STC}, \mathrm{G}_{\mathrm{STC}}=1000 \mathrm{~W} / \mathrm{m}^{2}\right.$ and $\mathrm{T}_{\mathrm{STC}}=25^{\circ} \mathrm{C}$ ): $\mathrm{P}_{\mathrm{MPP}}=154.905 \mathrm{~W} ; \mathrm{V}_{\mathrm{oc}}=41.4 \mathrm{~V}$; $\mathrm{V}_{\mathrm{MPP}}=34.5 \mathrm{~V} ; \mathrm{I}_{\mathrm{SC}}=5.03 \mathrm{~A} ; \mathrm{I}_{\mathrm{MPP}}=4.49 \mathrm{~A}$. The PV-curves for this panel under the static irradiances described in the EN505030:2010 standard are shown in Figure 8. The dashed red line shows the theoretical MPP for every irradiance value between $100 \mathrm{~W} / \mathrm{m}^{2}$ and $1100 \mathrm{~W} / \mathrm{m}^{2}$. The duty cycle-voltage curves for these same irradiances are plotted in Figure 9. It can be observed that there is a rise in PV voltage at the boost converter input with an increase in irradiance at the same duty cycle. This phenomenon is due to parasitic resistances in the simulated boost converter that cause a larger effect as the current produced by panel increases. 
Table 2. Irradiance profile as tested.

\begin{tabular}{|c|c|c|c|c|c|c|c|}
\hline \multicolumn{8}{|c|}{ a. Irradiance low to medium $\left(100 \mathrm{~W} / \mathrm{m}^{2}-500 \mathrm{~W} / \mathrm{m}^{2}\right)$} \\
\hline $\begin{array}{l}\text { Settling } \\
{[\mathrm{s}]}\end{array}$ & $\begin{array}{l}\text { Rep. } \\
{[\mathrm{n}]}\end{array}$ & $\begin{array}{c}\text { Slope } \\
{\left[\mathrm{W} / \mathrm{m}^{2} / \mathrm{s}\right]}\end{array}$ & $\begin{array}{c}\text { Rise } \\
{[\mathrm{s}]}\end{array}$ & $\begin{array}{l}\text { Wait } \\
{[\mathrm{s}]}\end{array}$ & $\begin{array}{c}\text { Fall } \\
{[\mathrm{s}]}\end{array}$ & $\begin{array}{l}\text { Wait } \\
{[\mathrm{s}]}\end{array}$ & $\begin{array}{c}\text { Duration } \\
{[\mathrm{s}]}\end{array}$ \\
\hline 3 & 2 & 50 & 8 & 0.1 & 8 & 0.1 & 35.4 \\
\hline 3 & 2 & 100 & 4 & 0.1 & 4 & 0.1 & 19.4 \\
\hline 3 & 3 & 200 & 2 & 0.1 & 2 & 0.1 & 15.6 \\
\hline 3 & 4 & 300 & 1.33 & 0.1 & 1.33 & 0.1 & 14.44 \\
\hline 3 & 6 & 500 & 0.8 & 0.1 & 0.8 & 0.1 & 13.8 \\
\hline 3 & 8 & 700 & 0.57 & 0.1 & 0.57 & 0.1 & 13.72 \\
\hline 3 & 10 & 1000 & 0.40 & 0.1 & 0.40 & 0.1 & 13 \\
\hline 3 & 10 & 1400 & 0.29 & 0.1 & 0.29 & 0.1 & 10.8 \\
\hline 3 & 10 & 2000 & 0.2 & 0.1 & 0.2 & 0.1 & 9 \\
\hline 3 & 10 & 3000 & 0.13 & 0.1 & 0.13 & 0.1 & 7.6 \\
\hline 3 & 10 & 5000 & 0.08 & 0.1 & 0.08 & 0.1 & 6.6 \\
\hline & & & & & & Total & 159.36 \\
\hline & & & & & & & 00:02:39 \\
\hline \multicolumn{8}{|c|}{ b. Irradiance medium to high $\left(300 \mathrm{~W} / \mathrm{m}^{2}-1000 \mathrm{~W} / \mathrm{m}^{2}\right)$} \\
\hline $\begin{array}{c}\text { Settling } \\
{[\mathrm{s}]}\end{array}$ & $\begin{array}{l}\text { Rep. } \\
{[\mathrm{n}]}\end{array}$ & $\begin{array}{c}\text { Slope } \\
{\left[\mathrm{W} / \mathrm{m}^{2} / \mathrm{s}\right]}\end{array}$ & $\begin{array}{c}\text { Rise } \\
{[\mathrm{s}]}\end{array}$ & $\begin{array}{l}\text { Wait } \\
{[\mathrm{s}]}\end{array}$ & $\begin{array}{c}\text { Fall } \\
{[\mathrm{s}]}\end{array}$ & $\begin{array}{l}\text { Wait } \\
{[\mathrm{s}]}\end{array}$ & $\begin{array}{c}\text { Duration } \\
{[\mathrm{s}]}\end{array}$ \\
\hline 3 & 10 & 1000 & 0.7 & 10 & 0.7 & 0.1 & 19 \\
\hline 3 & 10 & 1400 & 0.5 & 10 & 0.5 & 0.1 & 15 \\
\hline 3 & 10 & 2000 & 0.35 & 10 & 0.35 & 0.1 & 12 \\
\hline 3 & 10 & 3000 & 0.23 & 10 & 0.23 & 0.1 & 9.6 \\
\hline 3 & 10 & 5000 & 0.14 & 10 & 0.14 & 0.1 & 7.8 \\
\hline \multirow[t]{3}{*}{3} & 10 & 10,000 & 0.07 & 10 & 0.07 & 0.1 & 6.4 \\
\hline & & & & & & Total & 69.8 \\
\hline & & & & & & & 00:01:09 \\
\hline
\end{tabular}

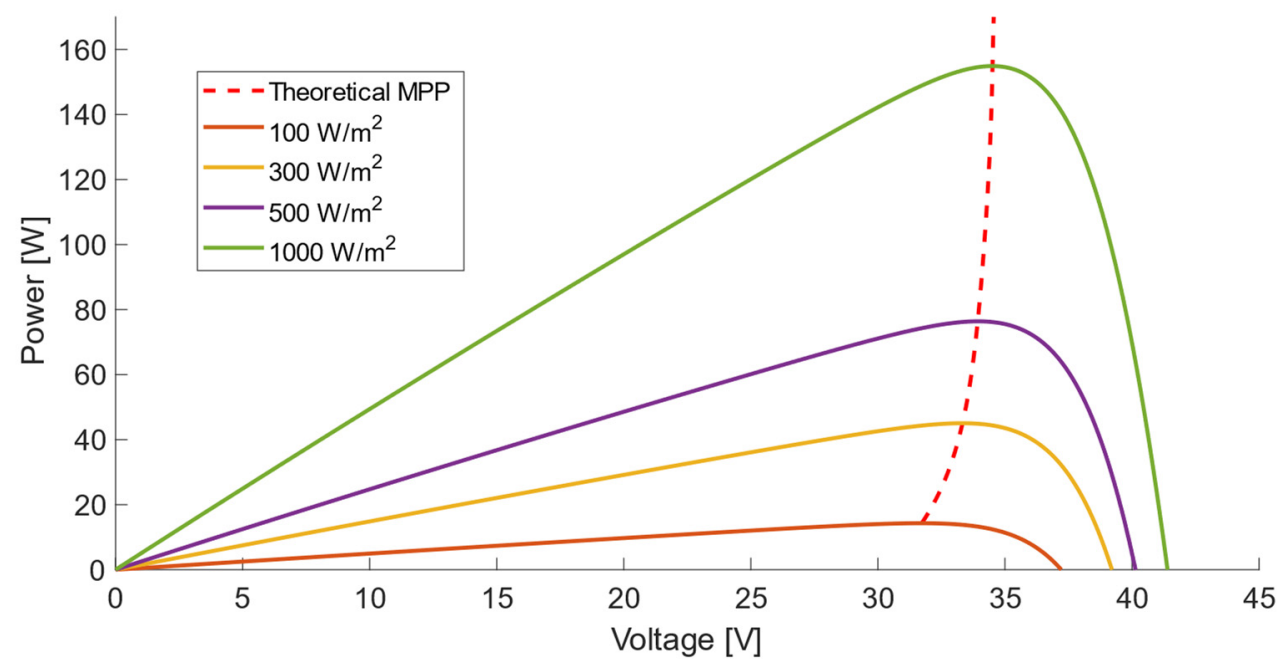

Figure 8. PV-curves for all static irradiance values described in the EN50530:2010 standard with the theoretical MPP of every intermediate irradiance for the solar panel, UP-M155M. 


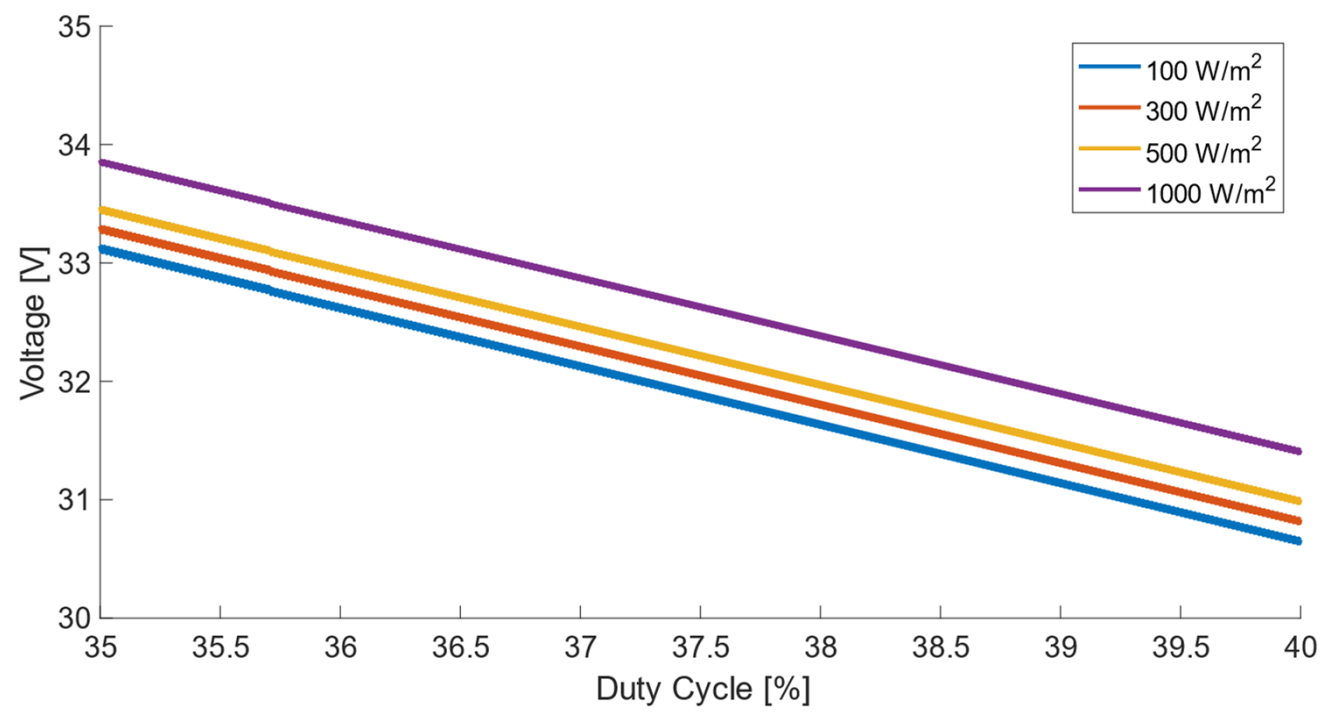

Figure 9. Simulated duty ratio-voltage curves of the boost converter and the solar panel UP-M155M curves for all static irradiance values described in the EN50530:2010 standard.

The overall efficiency during the test was calculated for both algorithms. Additionally, the dynamic efficiency for each set of iterations testing different slopes is calculated as follows:

$$
\% \text { Efficiency }=\frac{\int_{0}^{T_{M}} P}{\int_{0}^{T_{M}} P_{M P P}} \times 100,
$$

where $T_{M}$ consists of the simulation time, $P_{M P P}$ is the maximum theoretical power available at the MPP at the given conditions, and $P$ is the actual power delivered at the given operating point. The algorithms were tested with a fixed $T_{M P P T}=15 \mathrm{~ms}$. Parameter $\delta$ was also fixed size to $2 \%$ of the $\mathrm{V}_{\mathrm{oc}}$ as suggested in [8].

Additionally, the simulated results are compared to the theoretical $\mathrm{P}_{\mathrm{MPP}}$ and $\mathrm{V}_{\mathrm{MPP}}$. These comparisons will better illustrate contrasts between the algorithms being tested and the resulting efficiency differences. In Figure 10, the simulated behavior of both $\mathrm{P} \& \mathrm{O}$ and Td-MPPT while being subjected to a rise in irradiance from $100 \mathrm{~W} / \mathrm{m}^{2}$ to $1000 \mathrm{~W} / \mathrm{m}^{2}$ at a changing rate of $5000 \mathrm{~W} / \mathrm{m}^{2} / \mathrm{s}$ is displayed. This graphic format allows observing better the classical $\mathrm{P} \& \mathrm{O}$ drift, although it makes it harder to observe oscillations around MPP during static irradiance. It can be observed that both P\&O and Td-MPPT start around the theoretical MPP represented in the red dashed line. However, as MPP shifts towards the right with increasing irradiance, $\mathrm{P} \& \mathrm{O}$ keeps perturbing away from the optimal value until the perturbation produces a power reduction significant enough to counter the power increase caused by irradiance increases. In contrast, Td-MPPT tracks the theoretical MPP very close through its trajectory. 
(a) P\&O

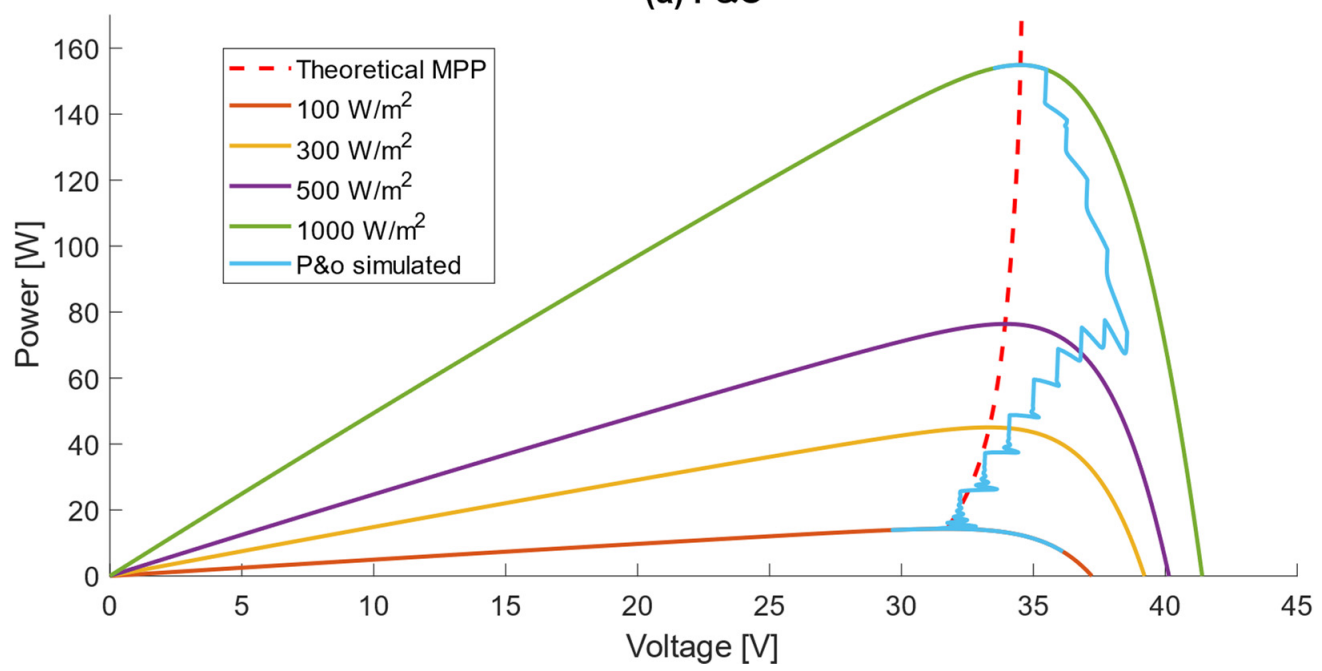

(b) Td-MPPT

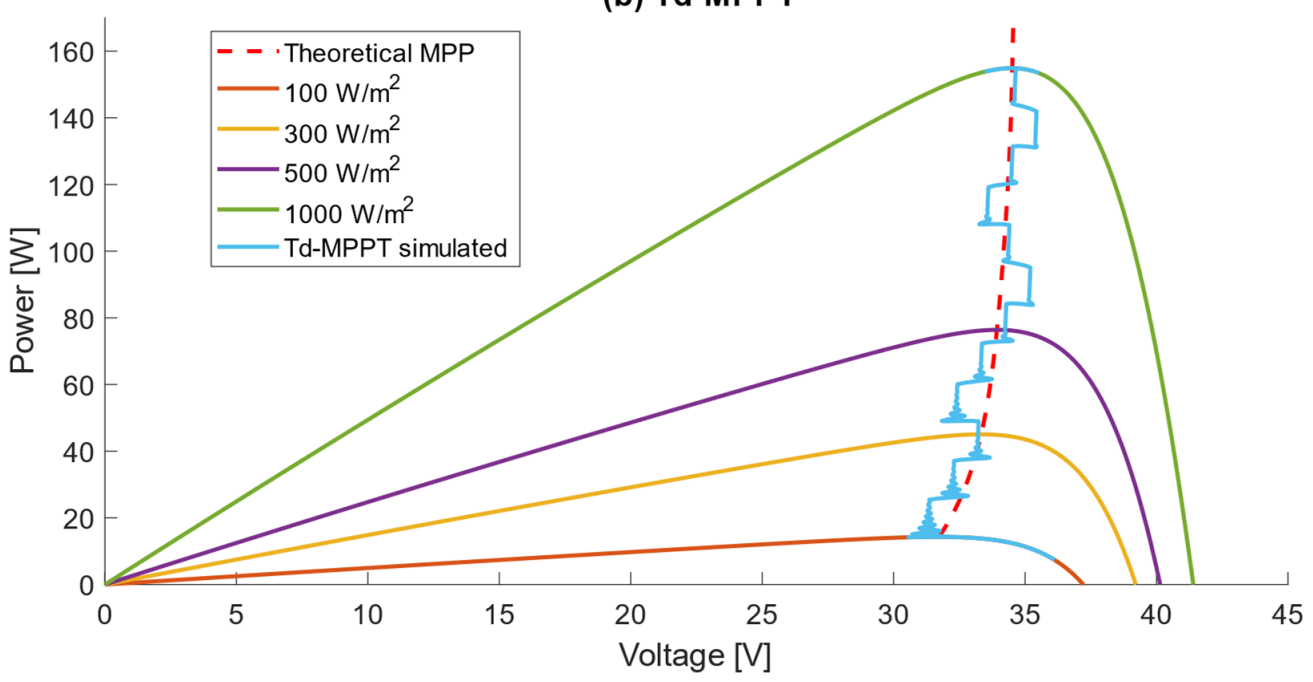

Figure 10. Simulated behavior of $\mathrm{P} \& \mathrm{O}(\mathbf{a})$ and Td-MPPT $(\mathbf{b})$ when subjected to a steady increase from $100 \mathrm{~W} / \mathrm{m}^{2}$ to $1000 \mathrm{~W} / \mathrm{m}^{2}$ at a changing rate of $5000 \mathrm{~W} / \mathrm{m}^{2} / \mathrm{s}$.

A format that includes time in the figure is presented from now on to better illustrate oscillations around the theoretical MPP. The classical P\&O behavior during the complete test described in Table 2 is shown in Figure 11a. The graph shows the simulated voltage and power (solid-blue) and the theoretical ideal at MPP (dotted-red). It can be noted that the algorithm tracks relatively well during the first iterations of both regions. In contrast, as the slopes become steeper in later iterations, the $\mathrm{P} \& \mathrm{O}$ displays significant drift from the MPP, negatively affecting the power output and, consequently, efficiency. It must be noted that the simulation results correspond with the behavior described in the literature $[13,19,35]$. The $\mathrm{dP}-\mathrm{P} \& \mathrm{O}$ in Figure 11b shows fewer and smaller excursions away from MPP than the P\&O. The proposed Td-MPPT displayed in Figure 11c does not exhibit drift issues and tracks the most effective during the complete test. Due to this, the overall efficiency of Td-MPPT reaches $99.68 \%$, while dP-P\&O achieves $99.56 \%$, and classical $\mathrm{P} \& \mathrm{O}$ drops to $97.26 \%$. 
(a) P\&O
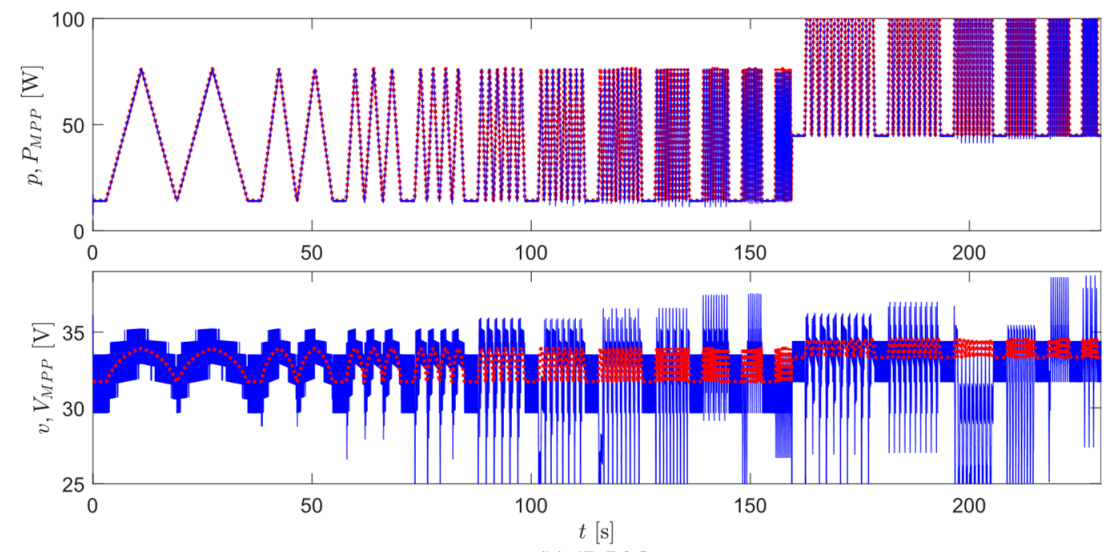

(b) $\mathrm{dP}-\mathrm{P} \& \mathrm{O}$
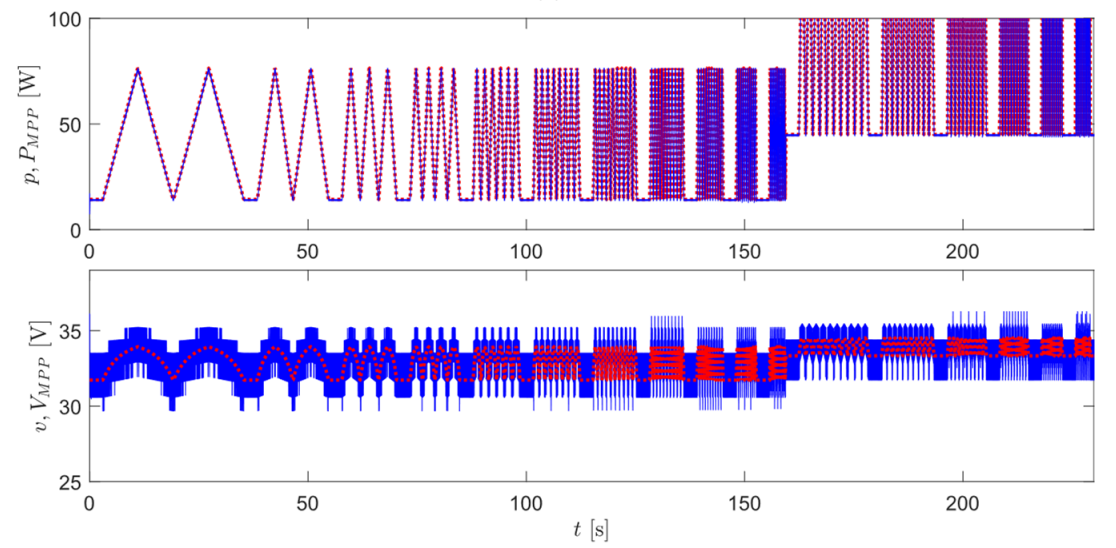

(c) Td-MPPT
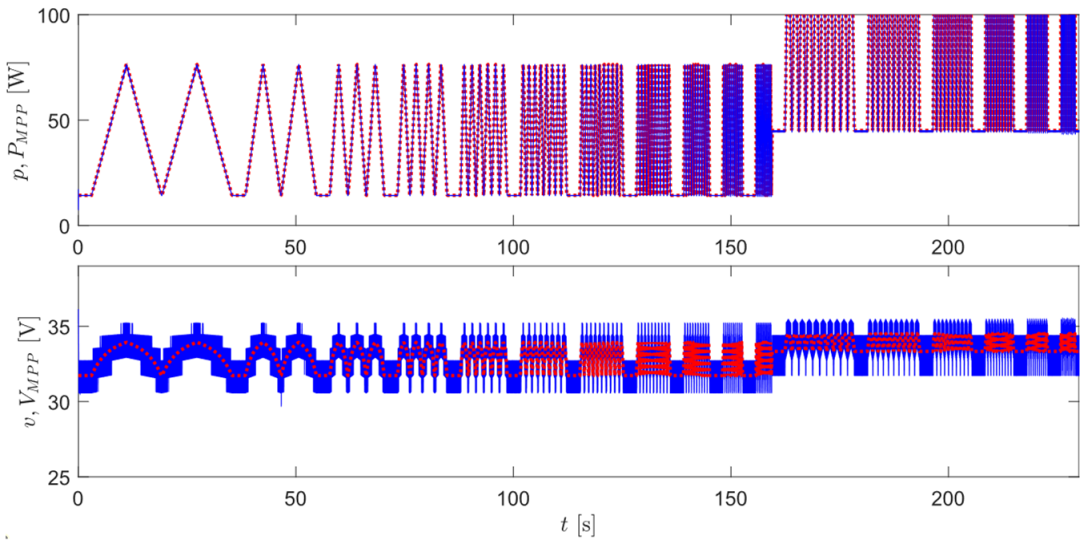

Figure 11. Behavior of the $\mathrm{P} \& \mathrm{O}(\mathbf{a}), \mathrm{dP}-\mathrm{P} \& \mathrm{O}(\mathbf{b})$ and Td-MPPT (c) during the full simulation: simulated PV voltage (solid-blue) and $\mathrm{V}_{\mathrm{MPP}}$ (dotted-red), corresponding PV power simulated (solid-blue), and $\mathrm{P}_{\mathrm{MPP}}$ (dotted-red).

Figure 12 shows the dynamic efficiency of the tested algorithms in response to the trapezoidal profiles of the test for the low to medium (solid-blue) and the medium to high (dashed-red) sections. It can be noted that $\mathrm{P} \& \mathrm{O}$ efficiency in Figure 12a deteriorates rapidly as the slope becomes steeper with each iteration. However, efficiency stops decaying after a certain point and rises with even steeper irradiance slopes. This phenomenon occurs because the $\mathrm{P} \& \mathrm{O}$ does not have enough time to drift away from the MPP with the fastest repeating trapezoidal irradiance profiles as with the slower ones. This contrast between fast $\left(700 \mathrm{~W} / \mathrm{m}^{2} / \mathrm{s}\right)$ and the fastest $\left(5000 \mathrm{~W} / \mathrm{m}^{2} / \mathrm{s}\right)$ irradiance slopes can be observed in Figure 13 . Similar results were observed in $[19,36]$ as it was noted that the $\mathrm{P} \& \mathrm{O}$ algorithm did not show significant drift from the MPP when being subjected to step-shaped irradiance changes. 
(a) P\&O

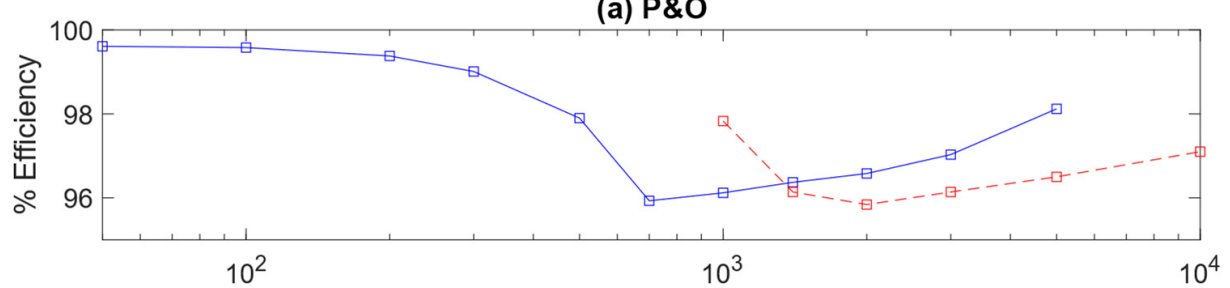

(b) dP-P\&O

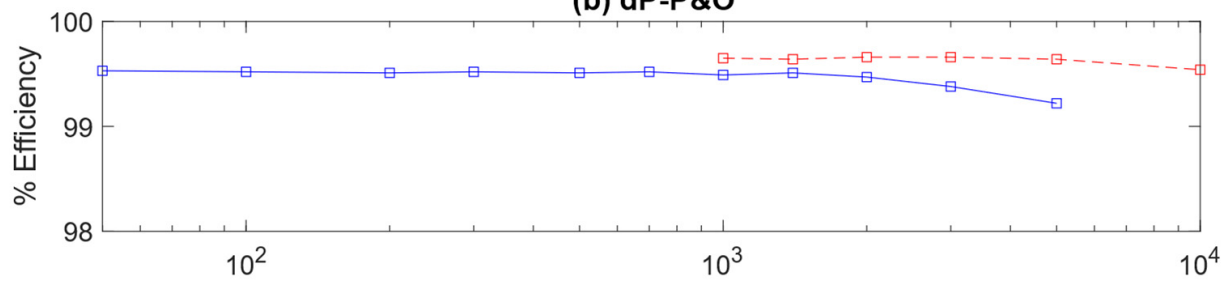

(c) Td-MPPT

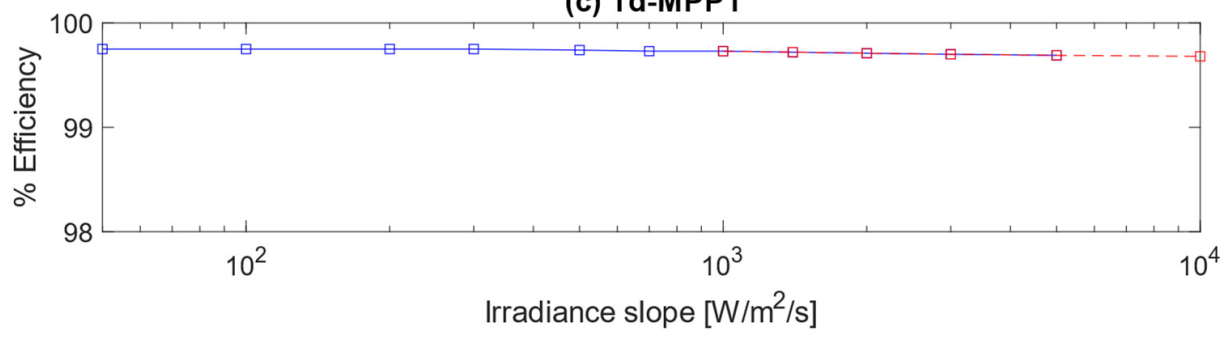

Figure 12. Plotted MPPT efficiency for every sequence of the trapezoidal shapes described in Table 2 organized according to the Irradiance slope of every section for the P\&O (a), dP-P\&O (b), and the Td-MPPT (c): efficiency for the low to medium irradiance test section (solid blue) and efficiency for the medium to high irradiance test section (dashed red).

(a) Irradiance slope $700 \mathrm{~W} / \mathrm{m}^{2} / \mathrm{s}$

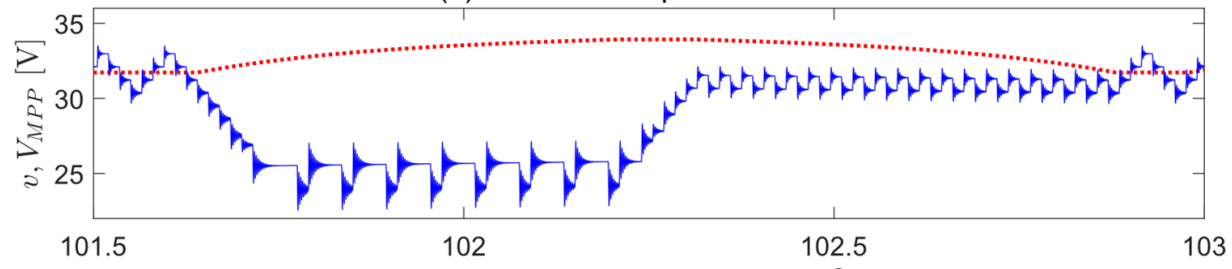

(b) Irradiance slope $5000 \mathrm{~W} / \mathrm{m}^{2} / \mathrm{s}$

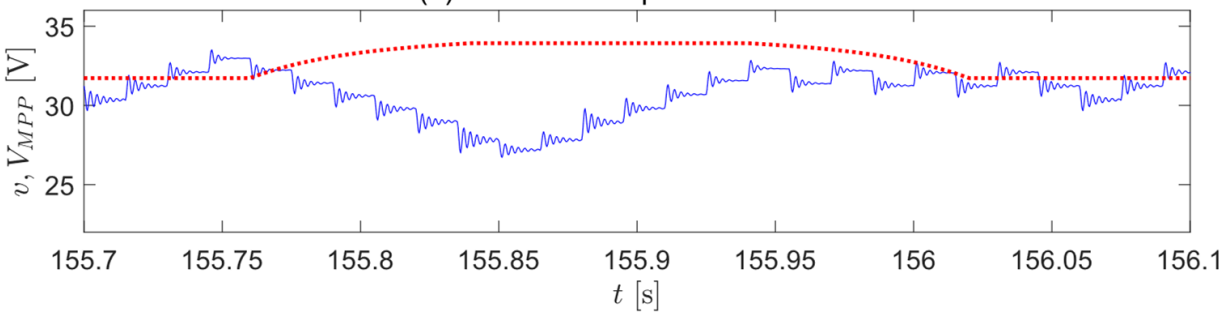

Figure 13. Behavior of the classical $\mathrm{P} \& \mathrm{O}$ during irradiance trapezoidal profiles tested with an irradiance slope of $700 \mathrm{~W} / \mathrm{m}^{2} / \mathrm{s}$ (a) and $5000 \mathrm{~W} / \mathrm{m}^{2} / \mathrm{s}$ (b); simulated PV voltage (solid-blue) and corresponding $\mathrm{V}_{\text {MPP }}$ (dotted-red).

It can be noted that the efficiency for $\mathrm{dP}-\mathrm{P} \& \mathrm{O}$ in Figure $12 \mathrm{~b}$ modification does not decay as fast with steeper slopes as with the classical P\&O. However, some degree of efficiency loss is displayed as the irradiance slope rises. Contrary to what is observed with these two algorithms, the proposed Td-MPPT in Figure 12c shows an almost imperceptible reduction in tracking efficiency even at the steepest irradiance slopes. It must be noted that the efficiency axis for both the $\mathrm{dP}-\mathrm{P} \& \mathrm{O}$ and the Td-MPPT are zoomed-in compared 
to the $\mathrm{P} \& \mathrm{O}$. This was used to observe the differences in efficiency between these two algorithms better.

In Figures 14-25, the response of the algorithms is displayed in more detail for some sections of the test or specific irradiance profiles. First, the case of the slightest slope $\left(50 \mathrm{~W} / \mathrm{m}^{2} / \mathrm{s}\right)$ of the first section (low to medium irradiance) is considered (Figures 14-16) as an example of the performance under a slowly changing rate of irradiance. Second, the fastest-changing rate $\left(5000 \mathrm{~W} / \mathrm{m}^{2} / \mathrm{s}\right)$ of the first test section (low to medium irradiance) is displayed (Figures 17-19) as an example of the performance under a very rapidly changing rate of irradiance. Then, a steady irradiance $\left(300 \mathrm{~W} / \mathrm{m}^{2}\right)$ case is considered to validate the steady-state oscillations in Figures 20-22. Lastly, a specific irradiance profile is proposed that takes irradiance down from a medium $\left(500 \mathrm{~W} / \mathrm{m}^{2}\right)$ to a low irradiance $\left(100 \mathrm{~W} / \mathrm{m}^{2}\right)$ at a fast-changing rate $\left(5000 \mathrm{~W} / \mathrm{m}^{2} / \mathrm{s}\right)$. This specific profile was designed to validate how the platform would react to irradiance on the PV panel being rapidly decreased by a passing obstacle.

(a)

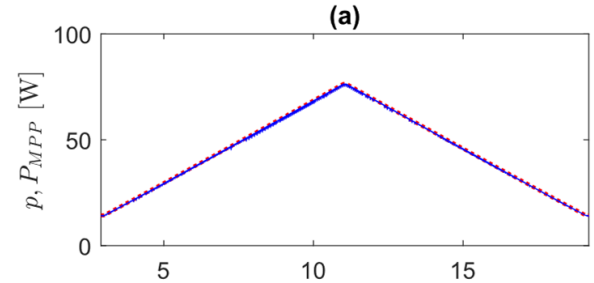

(c)

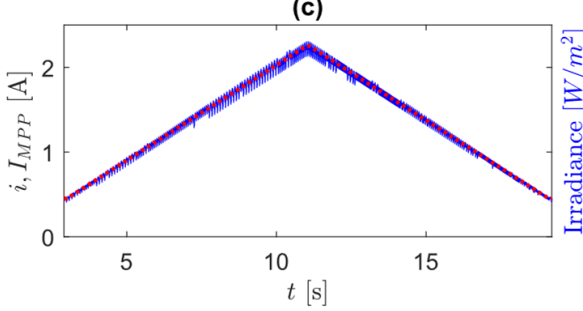

P\&O

(b)

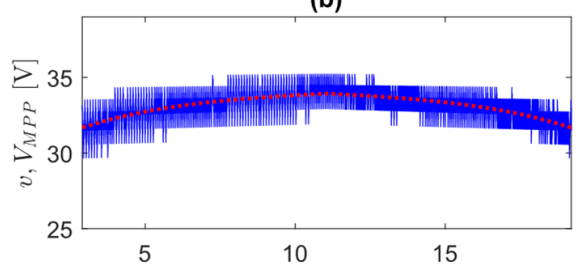

(d)

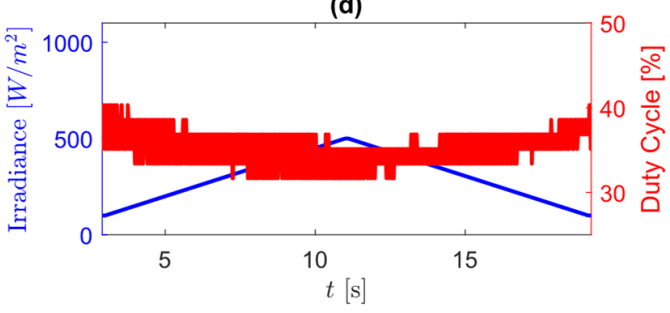

Figure 14. Response of the $\mathrm{P} \& \mathrm{O}$ algorithm to slow irradiance change $\left(50 \mathrm{~W} / \mathrm{m}^{2} / \mathrm{s}\right)$ from the low to medium irradiance section of the test; (a) Simulated PV power (solid-blue) and $\mathrm{P}_{\mathrm{MPP}}$ (dotted-red), (b) PV voltage simulated (solid-blue) and $\mathrm{V}_{\mathrm{MPP}}$ (dotted-red), (c) simulated PV current (solid-blue) and $\mathrm{I}_{\mathrm{MPP}}$ (dotted-red), and (d) irradiance (solid blue) and duty cycle (solid red).

dP-P\&O

(a)

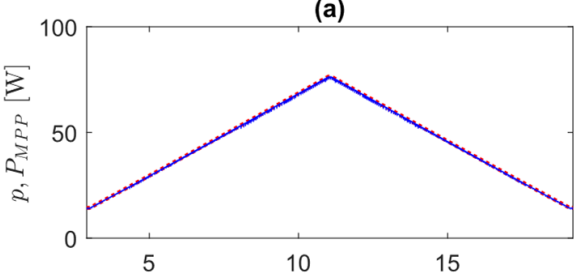

(c)

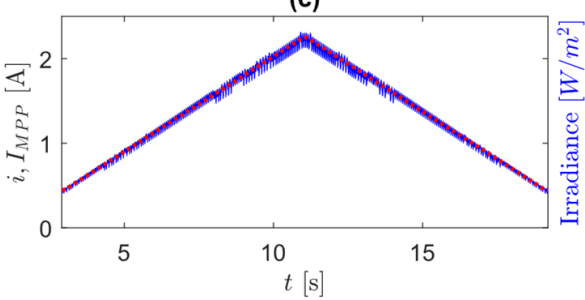

(b)

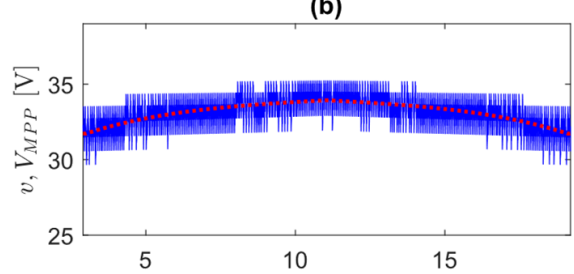

(d)

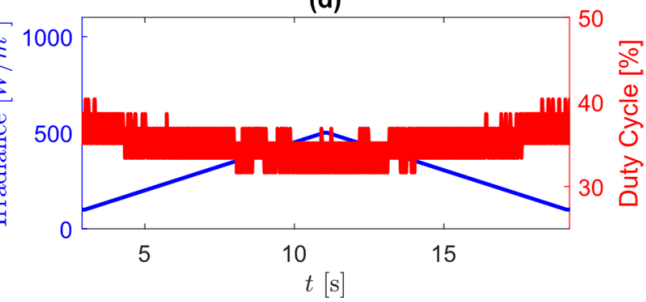

Figure 15. Response of the $\mathrm{dP}-\mathrm{P} \& \mathrm{O}$ algorithm to slow irradiance change $\left(50 \mathrm{~W} / \mathrm{m}^{2} / \mathrm{s}\right)$ from the low to medium irradiance section of the test; (a) Simulated PV power (solid-blue) and $\mathrm{P}_{\mathrm{MPP}}$ (dotted-red), (b) PV voltage simulated (solid-blue) and $\mathrm{V}_{\mathrm{MPP}}$ (dotted-red), (c) simulated PV current (solid-blue) and $\mathrm{I}_{\mathrm{MPP}}$ (dotted-red), and (d) irradiance (solid blue) and duty cycle (solid red). 


\section{Td-MPPT}

(a)

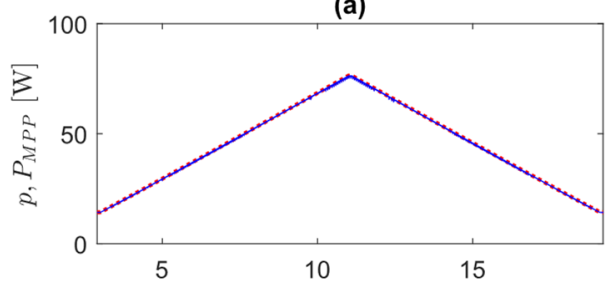

(c)

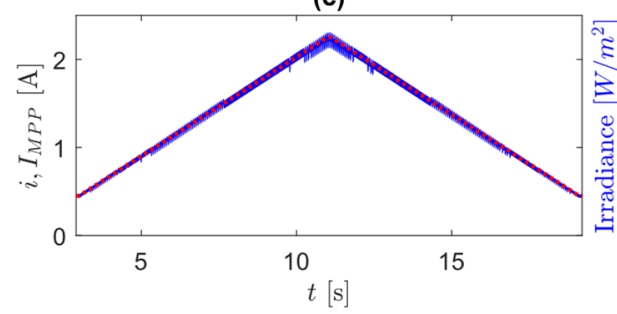

(b)

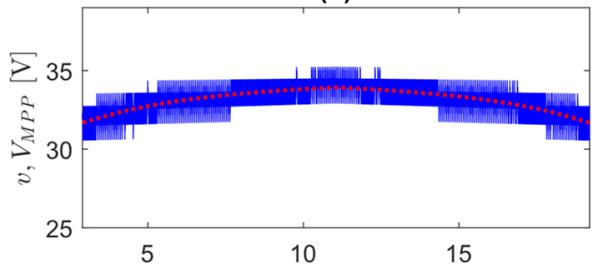

(d)

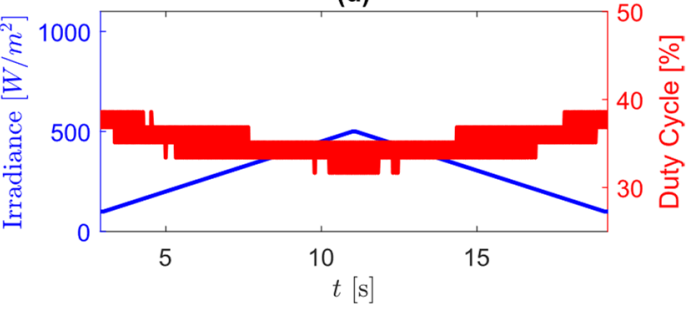

Figure 16. Response of the Td-MPPT algorithm to slow irradiance change $\left(50 \mathrm{~W} / \mathrm{m}^{2} / \mathrm{s}\right)$ from the low to medium irradiance section of the test; (a) Simulated PV power (solid-blue) and $\mathrm{P}_{\text {MPP }}$ (dotted-red), (b) PV voltage simulated (solid-blue) and $\mathrm{V}_{\mathrm{MPP}}$ (dotted-red), (c) simulated PV current (solid-blue) and $\mathrm{I}_{\mathrm{MPP}}$ (dotted-red), and (d) irradiance (solid blue) and duty cycle (solid red).

In Figures 14-16 it can be noted that both algorithms track the MPPT (dotted-red) very similarly for the slightest slope scenario. During this test section, the P\&O (Figure 14) obtained an efficiency of 99.61\%, the dP-P\&O (Figure 15) achieved 99.53\%, and lastly, Td-MPPT (Figure 16) reached the highest efficiency at $99.75 \%$.

P\&O

(a)

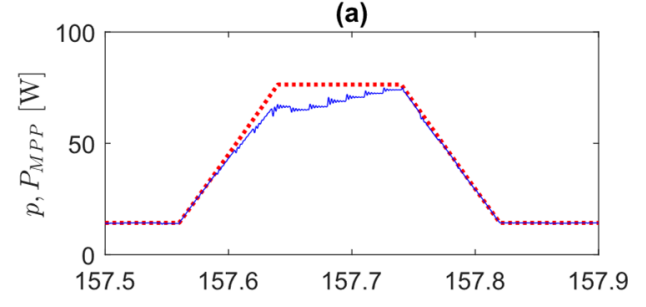

(c)

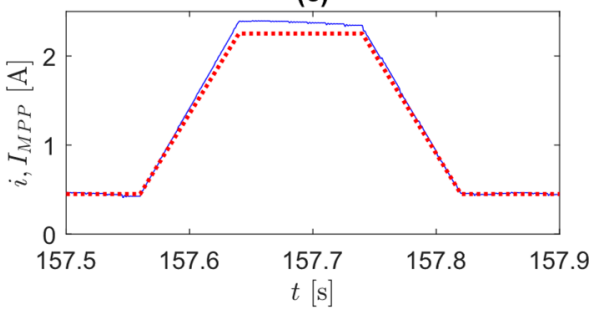

(b)

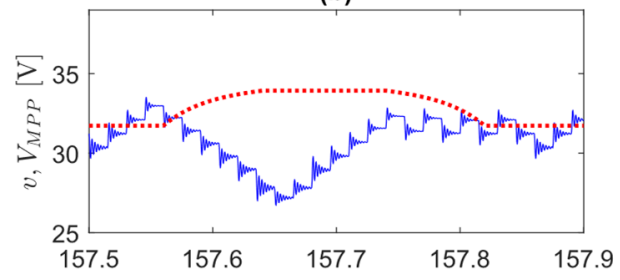

(d)

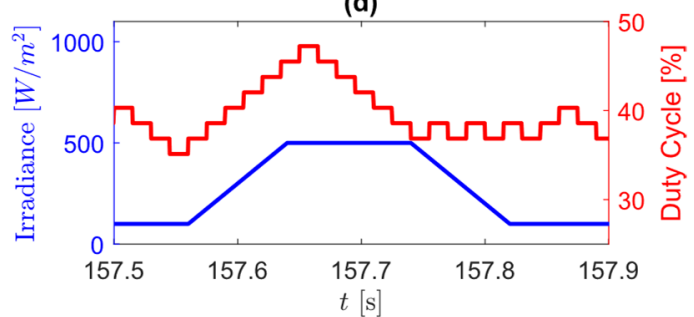

Figure 17. Response of the $\mathrm{P} \& \mathrm{O}$ algorithm to fast irradiance change $\left(5000 \mathrm{~W} / \mathrm{m}^{2} / \mathrm{s}\right)$ from the low to medium irradiance section of the test; (a) Simulated PV power (solid-blue) and PMPP (dotted-red), (b) PV voltage simulated (solid-blue) and $\mathrm{V}_{\mathrm{MPP}}$ (dotted-red), (c) simulated PV current (solid-blue) and $\mathrm{I}_{\mathrm{MPP}}$ (dotted-red), and (d) irradiance (solid blue) and duty cycle (solid red).

For the fastest irradiance changes in the low to medium section of the test, it can be observed that the P\&O (Figure 17) shows the characteristic drift reported in the literature. The operating point determined by the MPPT (As in Equation (10)) can be observed in Figure 17a. Even when MPPT perturbs in the correct direction at the beginning of the trapezoidal form, it cannot detect that it has passed the MPP. Furthermore, when the irradiance profile begins its descent, the $\mathrm{P} \& \mathrm{O}$ attributes the measured power reduction to the effect of the perturbations introduced and keeps perturbing around the same operating point until irradiance reaches a steady state. In Figure $17 c$, it can be observed that the 
current of the system drops under the theoretical maximum because of this behavior. Also, this produces a decrease in the power output that can be noted in Figure 17a. Because of this, $\mathrm{P} \& \mathrm{O}$ reaches an efficiency of $98.12 \%$ (average). On the other hand, dP-P\&O (Figure 18) becomes confused at the beginning of the trapezoid and corrects the search direction by reaching MPP shortly after. The efficiency achieved by it during this test was $99.22 \%$.

In contrast, the Td-MPPT (Figure 19) keeps perturbing in the correct direction almost every time, and the actual operation point (solid-blue) does not drift away from MPP. The system only becomes confused when the irradiance changing rate shows a significant enough change, i.e., when the edges of the trapezoidal irradiance profile occur between a cycle of the MPPT. However, the system corrects the trajectory and effectively follows MPP. Because of this, the efficiency of Td-MPPT for this section is $99.69 \%$ (average).

dP-P\&O

(a)

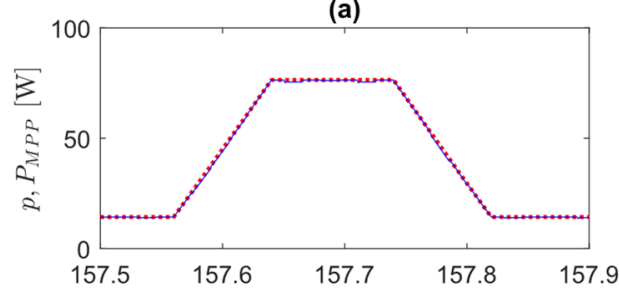

(c)

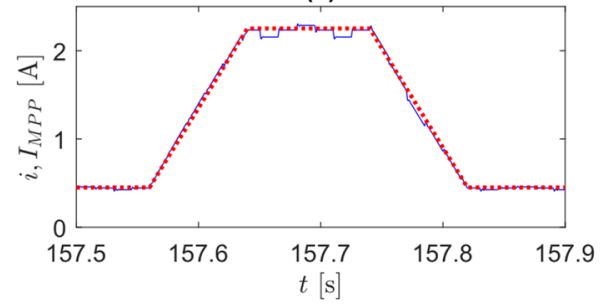

(b)

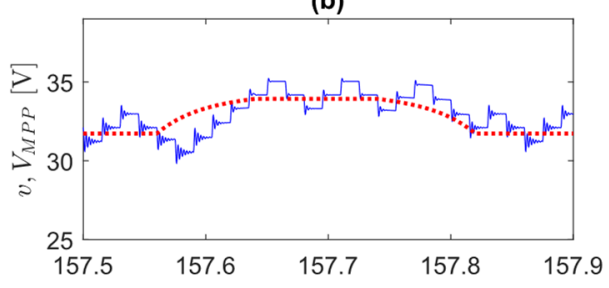

(d)

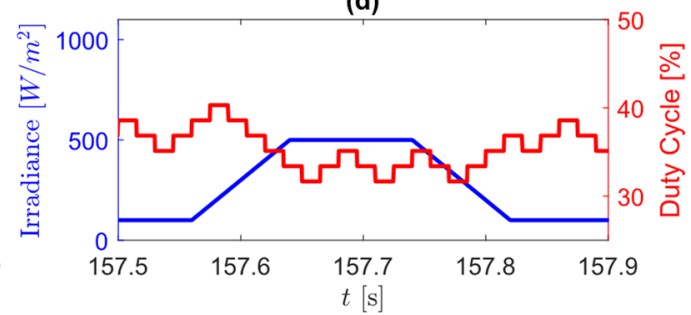

Figure 18. Response of the dP-P\&O algorithm to fast irradiance change $\left(5000 \mathrm{~W} / \mathrm{m}^{2} / \mathrm{s}\right)$ from the low to medium irradiance section of the test; (a) Simulated PV power (solid-blue) and $\mathrm{P}_{\mathrm{MPP}}$ (dotted-red), (b) PV voltage simulated (solid-blue) and $\mathrm{V}_{\mathrm{MPP}}$ (dotted-red), (c) simulated PV current (solid-blue) and $\mathrm{I}_{\mathrm{MPP}}$ (dotted-red), and (d) irradiance (solid blue) and duty cycle (solid red).

(a)

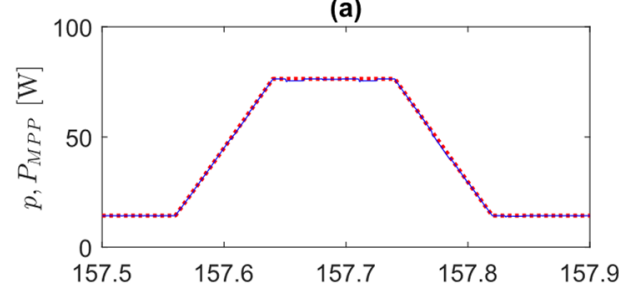

(c)

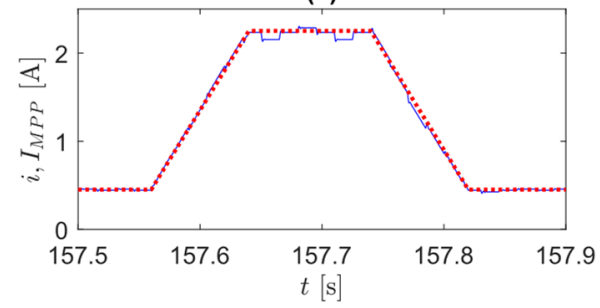

Td-MPPT

(b)

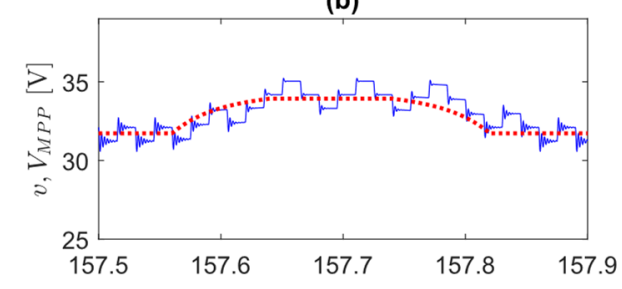

(d)

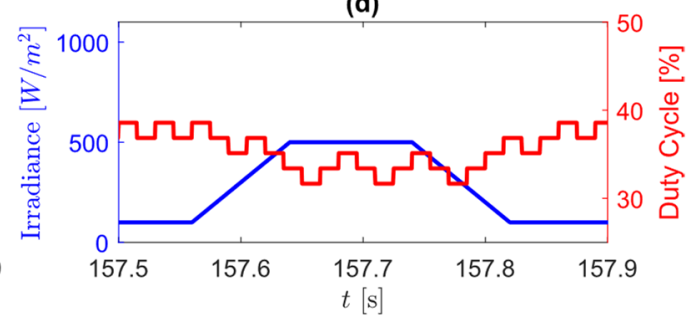

Figure 19. Response of the Td-MPPT algorithm to fast irradiance change $\left(5000 \mathrm{~W} / \mathrm{m}^{2} / \mathrm{s}\right)$ from the low to medium irradiance section of the test; (a) Simulated PV power (solid-blue) and P $\mathrm{MPP}$ (dotted-red), (b) PV voltage simulated (solid-blue) and $\mathrm{V}_{\mathrm{MPP}}$ (dotted-red), (c) simulated PV current (solid-blue) and $\mathrm{I}_{\mathrm{MPP}}$ (dotted-red), and (d) irradiance (solid blue) and duty cycle (solid red). 
Lastly, the behavior of the algorithms during steady irradiance of $300 \mathrm{~W} / \mathrm{m}^{2}$ is displayed in Figure 16. It can be observed that both P\&O (Figure 16a) has to perturb three times around the MPP while dP-P\&O (Figure 16b) performs only two. This difference could be caused by the fact that $\mathrm{PP}-\mathrm{P} \& \mathrm{O}$ obtains additional information at $T_{M P P T} / 2$ since the system has not reached a steady state when it is being measured. In contrast, Td-MPPT (Figure 16c) perturbs only one time in the same direction, reducing steady-state oscillations. Accordingly, $\mathrm{P} \& \mathrm{O}$ achieved $99.35 \%$ and $\mathrm{dP}-\mathrm{P} \& \mathrm{O}$ achieved $99.38 \%$ efficiency during the steady irradiance settling time ( $3 \mathrm{~s})$ of the first part of the test. In comparison, Td-MPPT (99.42) achieved $99.82 \%$ efficiency.

$\mathrm{P} \& \mathrm{O}$

(a)

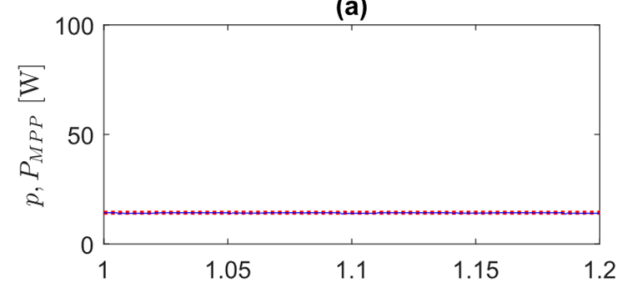

(c)

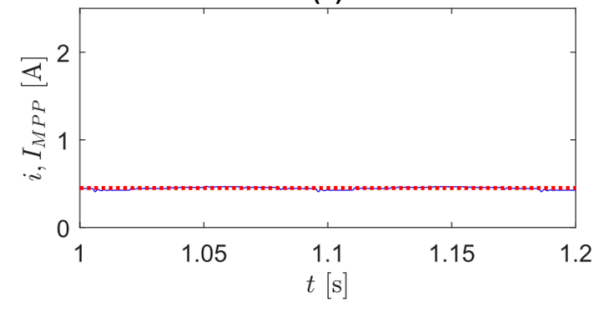

(b)

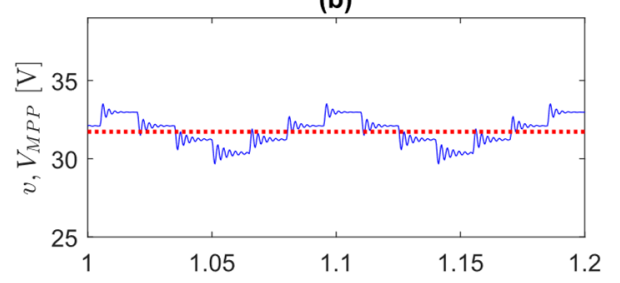

(d)

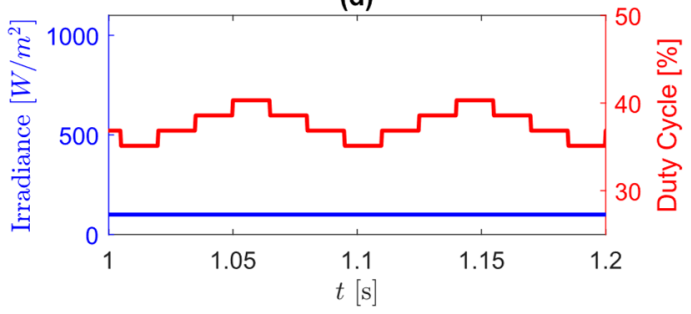

Figure 20. Response of the $\mathrm{P} \& \mathrm{O}$ algorithm to steady irradiance at $\left(300 \mathrm{~W} / \mathrm{m}^{2}\right)$; (a) Simulated PV power (solid-blue) and $\mathrm{P}_{\text {MPP }}$ (dotted-red), (b) PV voltage simulated (solid-blue) and $\mathrm{V}_{\text {MPP }}$ (dottedred), (c) simulated PV current (solid-blue) and $\mathrm{I}_{\text {MPP }}$ (dotted-red), and (d) irradiance (solid blue) and duty cycle (solid red).

dP-P\&O

(a)

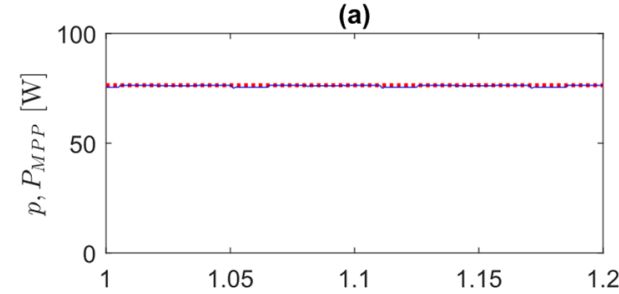

(b)

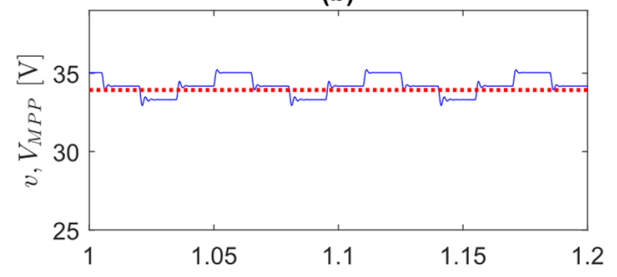

(c)

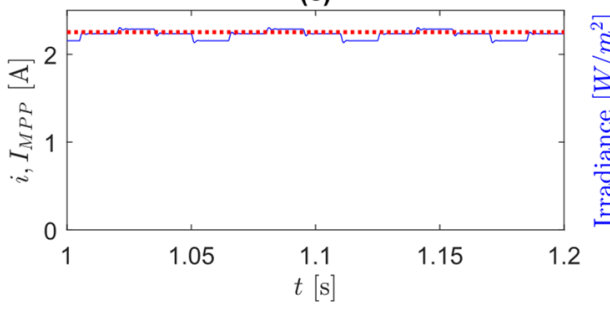

(d)

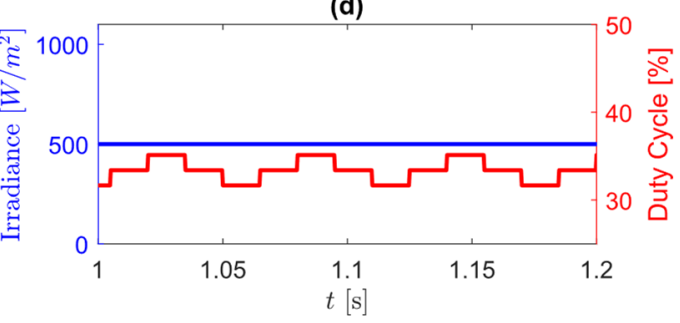

Figure 21. Response of the $\mathrm{dP}-\mathrm{P} \& \mathrm{O}$ algorithm to steady irradiance at $\left(300 \mathrm{~W} / \mathrm{m}^{2}\right)$; (a) Simulated PV power (solid-blue) and $\mathrm{P}_{\mathrm{MPP}}$ (dotted-red), (b) PV voltage simulated (solid-blue) and $\mathrm{V}_{\mathrm{MPP}}$ (dottedred), (c) simulated PV current (solid-blue) and $\mathrm{I}_{\mathrm{MPP}}$ (dotted-red), and (d) irradiance (solid blue) and duty cycle (solid red). 
(a)

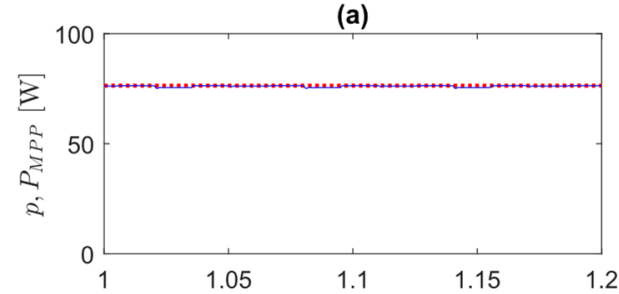

(c)

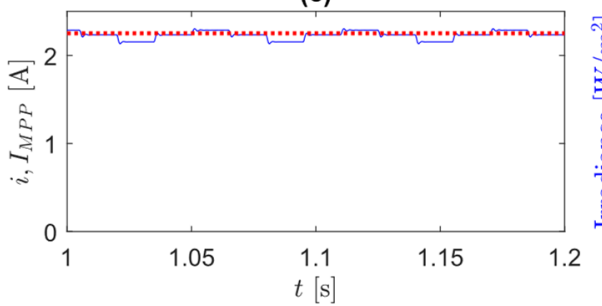

(b)

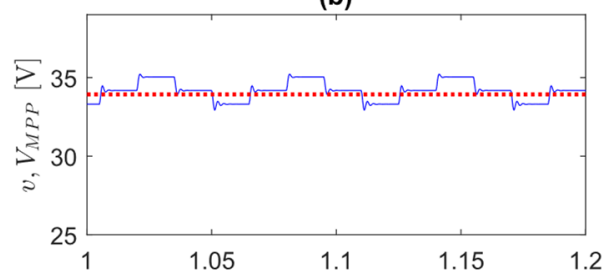

(d)

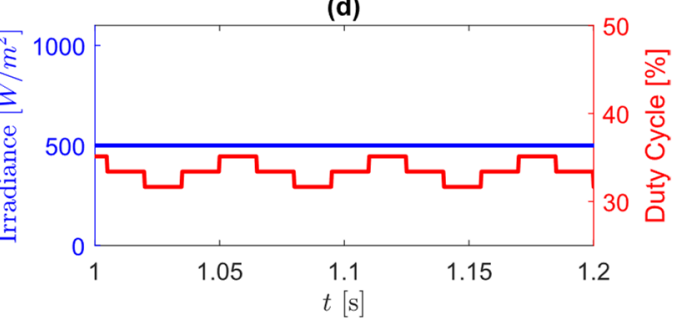

Figure 22. Response of the Td-MPPT algorithm to steady irradiance at $\left(300 \mathrm{~W} / \mathrm{m}^{2}\right)$; (a) Simulated PV power (solid-blue) and $\mathrm{P}_{\text {MPP }}$ (dotted-red), (b) PV voltage simulated (solid-blue) and $\mathrm{V}_{\text {MPP }}$ (dottedred), (c) simulated PV current (solid-blue) and $\mathrm{I}_{\text {MPP }}$ (dotted-red), and (d) irradiance (solid blue) and duty cycle (solid red).

(a)

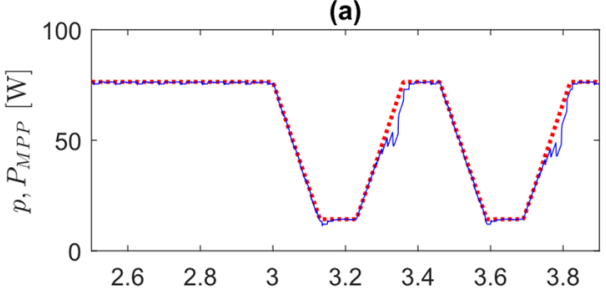

(c)

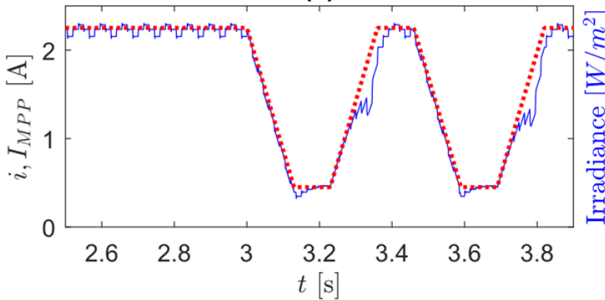

P\&O

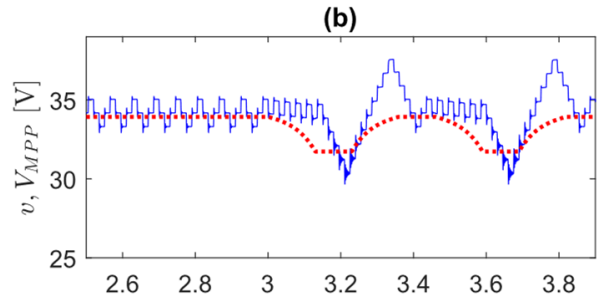

(d)

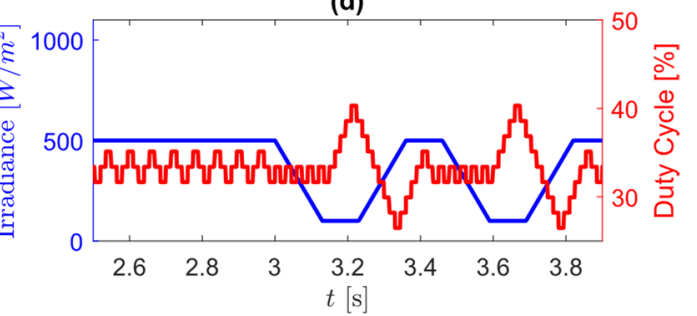

Figure 23. Response of the $\mathrm{P} \& \mathrm{O}$ algorithm to fast irradiance change $\left(5000 \mathrm{~W} / \mathrm{m}^{2} / \mathrm{s}\right)$ from medium $\left(500 \mathrm{~W} / \mathrm{m}^{2}\right)$ to low $\left(100 \mathrm{~W} / \mathrm{m}^{2}\right)$ irradiance and back; (a) Simulated PV power (solid-blue) and $\mathrm{P}_{\mathrm{MPP}}$ (dotted-red), (b) PV voltage simulated (solid-blue) and $\mathrm{V}_{\text {MPP }}$ (dotted-red), (c) simulated PV current (solid-blue) and $\mathrm{I}_{\mathrm{MPP}}$ (dotted-red), and (d) irradiance (solid blue) and duty cycle (solid red).

For the behavior of the algorithms during steady irradiance of $300 \mathrm{~W} / \mathrm{m}^{2}$ displayed in Figures 20-22, the P\&O (Figure 20) has to perturb three times around the MPP, while the $\mathrm{dP}-\mathrm{P} \& \mathrm{O}$ (Figure 21) performs only two. This difference could be caused by the fact that $\mathrm{dP}-$ $\mathrm{P} \& \mathrm{O}$ obtains additional information at $T_{M P P T} / 2$ since the system has not reached a steady state when it is being measured. In contrast, Td-MPPT (Figure 22) perturbs only one time in the same direction, reducing steady-state oscillations. Accordingly, during the steady irradiance settling time (3 s) of the first part of the test, the P\&O obtained $99.35 \%$ efficiency, the dP-P\&O achieved 99.38\% efficiency, and the Td-MPPT reached $99.82 \%$ efficiency. 
dP-P\&O

(a)

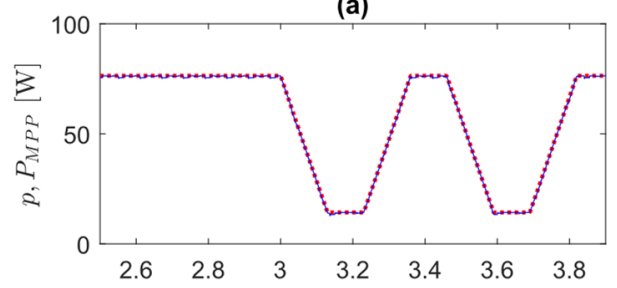

(c)

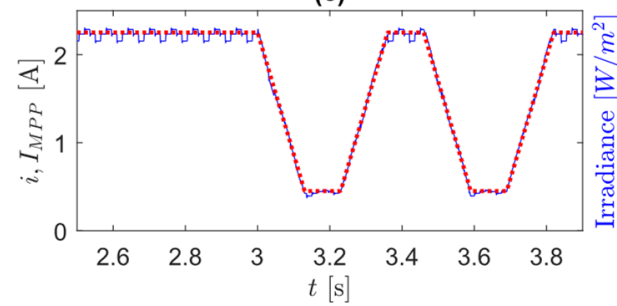

(b)

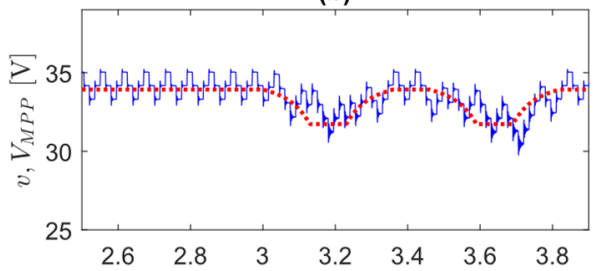

(d)

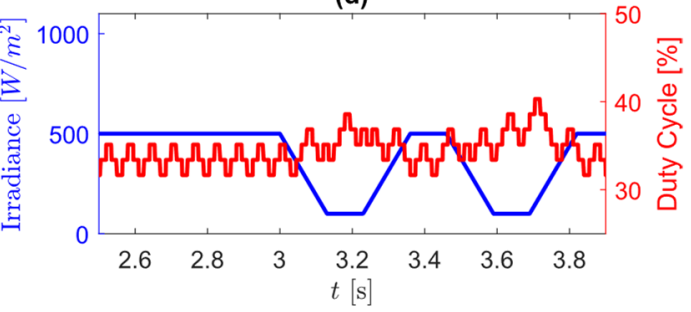

Figure 24. Response of the $\mathrm{dP}-\mathrm{P} \& \mathrm{O}$ algorithm to fast irradiance change $\left(5000 \mathrm{~W} / \mathrm{m}^{2} / \mathrm{s}\right)$ from medium $\left(500 \mathrm{~W} / \mathrm{m}^{2}\right)$ to low $\left(100 \mathrm{~W} / \mathrm{m}^{2}\right)$ irradiance and back; (a) Simulated PV power (solid-blue) and $\mathrm{P}_{\mathrm{MPP}}$ (dotted-red), (b) PV voltage simulated (solid-blue) and $\mathrm{V}_{\mathrm{MPP}}$ (dotted-red), (c) simulated PV current (solid-blue) and $\mathrm{I}_{\text {MPP }}$ (dotted-red), and (d) irradiance (solid blue) and duty cycle (solid red).

Td-MPPT

(a)

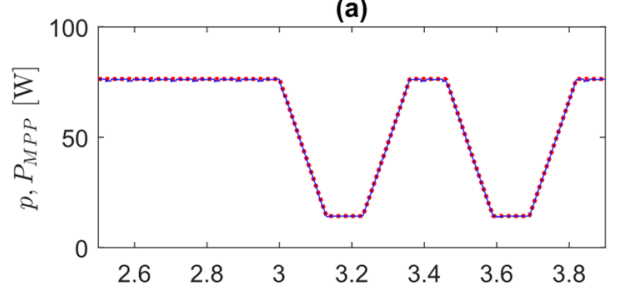

(c)

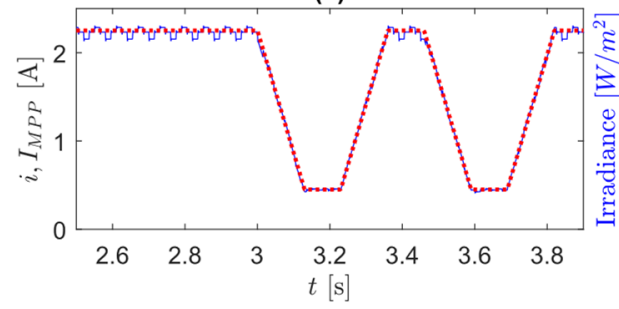

(b)

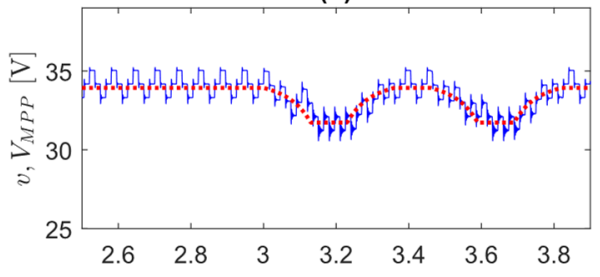

(d)

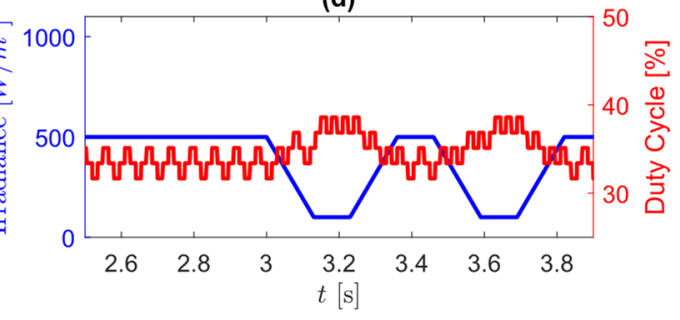

Figure 25. Response of the Td-MPPT algorithm to fast irradiance change (5000 W/ $\left.\mathrm{m}^{2} / \mathrm{s}\right)$ from medium $\left(500 \mathrm{~W} / \mathrm{m}^{2}\right)$ to low $\left(100 \mathrm{~W} / \mathrm{m}^{2}\right)$ irradiance and back; (a) Simulated PV power (solid-blue) and $\mathrm{P}_{\mathrm{MPP}}$ (dotted-red), (b) PV voltage simulated (solid-blue) and $\mathrm{V}_{\mathrm{MPP}}$ (dotted-red), (c) simulated PV current (solid-blue) and $\mathrm{I}_{\text {MPP }}$ (dotted-red), and (d) irradiance (solid blue) and duty cycle (solid red).

In Figures 23-25, inverted profiles proceeding from $500 \mathrm{~W} / \mathrm{m}^{2}$ to $100 \mathrm{~W} / \mathrm{m}^{2}$ at a change rate of $5000 \mathrm{~W} / \mathrm{m}^{2} / \mathrm{s}$ were tested for the three algorithms proposed. This change rate is the same as the fastest presented in the EN50530 test (Figures 17-19). However, during this test the irradiance starts at a medium value $\left(500 \mathrm{~W} / \mathrm{m}^{2}\right)$ and drops to a low value $\left(100 \mathrm{~W} / \mathrm{m}^{2}\right)$. A profile such as this could be observed if irradiance is blocked by an obstacle, e.g., a moving cloud. It can be observed that the P\&O (Figure 23) becomes confused only when irradiance rises from a low to a medium value. This simulation is consistent with the behavior observed in the literature $[1,2,19]$. The efficiency of the $\mathrm{P} \& \mathrm{O}$ was similar to the standard test with a changing rate of $5000 \mathrm{~W} / \mathrm{m}^{2} / \mathrm{s}(98.05 \%)$. The $\mathrm{dP}-\mathrm{P} \& \mathrm{O}$ (Figure 24) displayed a better efficiency under this test than under the standard one (99.39\%). Moreover, Td-MPPT (Figure 25) showed a slightly lower efficiency than the observed under the standard test $(99.59 \%)$. It must be noted that these results were very 
similar to the ones observed in Figures 23-25, with the P\&O showing the worst performance of the three algorithms tested and Td-MPPT performing the best.

Partial shading is not addressed in this work since it would need a different approach and methodology since EN50530 only considers uniform irradiance in the test ramps. However, a modification to Td-MPPT would be relatively simple. For example, in [37], the authors propose a novel probability algorithm to track the global maximum power point (GMPP), and then a variable step-size $\mathrm{P} \& \mathrm{O}$ performs fine tracking.

\section{Conclusions}

This paper presents a novel MPPT algorithm referred to as Td-MPPT based on the classical $\mathrm{P} \& \mathrm{O}$ algorithm. This algorithm calculates the derivative of power during transient states of an undamped controller to reduce the number of oscillations around the MPP under steady-state irradiance. In this manner, it determines whether a perturbation was performed in the correct direction. Since the algorithm proposed is based on a conventional MPPT, the complexity and hardware implementation are relatively simple compared to other algorithms. Because of this, the implementation ease and cost of the proposed algorithm are reduced. Although the tracking speed of these algorithms is traditionally low, simulations showed that the Td-MPPT algorithm efficiently tracked MPP during a test based on the EN50530 standard multiplied by a factor of $10^{2}$. An overall efficiency improvement over the algorithms benchmarked was observed for steady-state irradiance and rapid irradiation changes. Since the algorithm is based on a hill-climbing method, it is possible to combine it with partial shading techniques to address this issue. Furthermore, the presented method could be easily extended or mixed with variable perturbation times and perturbation sizes techniques already being applied to the classical $\mathrm{P} \& \mathrm{O}$ to improve its performance further. It was observed that an approximation of the natural frequency could be used to tune the proposed algorithm. However, this could not be optimal under every scenario. Because of this, the algorithm can be further improved by adding a self-tuning stage in the future. This modification could also facilitate the implementation of Td-MPPT for different kinds of topologies and PV-panel types. To this end, a common improvement to the classical P\&O is to combine it with more advanced methods to track GMPP. The same modifications could be easily applied to the proposed method to make it suitable to operate under partial shading conditions for future works.

Author Contributions: R.I.R.-C. participated in the literature review, methodology, formal analysis, laboratory test, and writing; L.J.R., conceptualization and supervision; A.B. and M.I.F.-B. participated in review and editing; A.Y.A. participated in data processing. All authors have read and agreed to the published version of the manuscript.

Funding: The first author would like to thank Consejo Nacional de Ciencia Tecnología (Av. Insurgentes Sur 1582, C.P. 03940, México. Phone Number: +52-(55)-5322-7700) (CONACYT) for doctoral grant 713240 .

Conflicts of Interest: The authors declare no conflict of interest. The funders had no role in the design of the study; in the collection, analyses, or interpretation of data; in the writing of the manuscript; or in the decision to publish the results.

\section{References}

1. Subudhi, B.; Pradhan, R. A Comparative Study on Maximum Power Point Tracking Techniques for Photovoltaic Power Systems. IEEE Trans. Sustain. Energy 2012, 4, 89-98. [CrossRef]

2. Karami, N.; Moubayed, N.; Outbib, R. General review and classification of different MPPT Techniques. Renew. Sustain. Energy Rev. 2017, 68, 1-18. [CrossRef]

3. Kandemir, E.; Cetin, N.S.; Borekci, S. A comprehensive overview of maximum power extraction methods for PV systems. Renew. Sustain. Energy Rev. 2017, 78, 93-112. [CrossRef]

4. Li, S.; Ping, A.; Liu, Y.; Ma, X.; Li, C. A variable-weather-parameter MPPT method based on a defined characteristic resistance of photovoltaic cell. Sol. Energy 2020, 199, 673-684. [CrossRef]

5. El Aamri, F.; Maker, H.; Sera, D.; Spataru, S.V.; Guerrero, J.M.; Mouhsen, A. A Direct Maximum Power Point Tracking Method for Single-Phase Grid-Connected PV Inverters. IEEE Trans. Power Electron. 2017, 33, 8961-8971. [CrossRef] 
6. Ahmed, J.; Salam, Z. A critical evaluation on maximum power point tracking methods for partial shading in PV systems. Renew. Sustain. Energy Rev. 2015, 47, 933-953. [CrossRef]

7. Elgendy, M.; Zahawi, B.; Atkinson, D. Evaluation of perturb and observe MPPT algorithm implementation techniques. In Proceedings of the 6th IET International Conference on Power Electronics, Machines and Drives (PEMD 2012), Bristol, UK, 27-29 March 2012; pp. 1-6. [CrossRef]

8. Pandey, A.; Dasgupta, N.; Mukerjee, A.K. High-Performance Algorithms for Drift Avoidance and Fast Tracking in Solar MPPT System. IEEE Trans. Energy Convers. 2008, 23, 681-689. [CrossRef]

9. Piegari, L.; Rizzo, R. Adaptive perturb and observe algorithm for photovoltaic maximum power point tracking. IET Renew. Power Gener. 2010, 4, 317-328. [CrossRef]

10. Kollimalla, S.K.; Mishra, M.K. Variable Perturbation Size Adaptive P\&O MPPT Algorithm for Sudden Changes in Irradiance. IEEE Trans. Sustain. Energy 2014, 5, 718-728. [CrossRef]

11. Xiao, W.; Dunford, W.G. A modified adaptive hill climbing MPPT method for photovoltaic power systems. In Proceedings of the 2004 IEEE 35th Annual Power Electronics Specialists Conference (IEEE Cat. No. 04CH37551), Aachen, Germany, 20-25 June 2004; pp. 1957-1963.

12. Kumar, V.; Ghosh, S.; Naidu, N.S.; Kamal, S.; Saket, R.; Nagar, S. Load voltage-based MPPT technique for standalone PV systems using adaptive step. Int. J. Electr. Power Energy Syst. 2021, 128, 106732. [CrossRef]

13. Bhattacharyya, S.; Kumar, P.D.S.; Samanta, S.; Mishra, S. Steady Output and Fast Tracking MPPT (SOFT-MPPT) for P\&O and InC Algorithms. IEEE Trans. Sustain. Energy 2021, 12, 293-302. [CrossRef]

14. Zhang, F.; Thanapalan, K.; Procter, A.; Carr, S.; Maddy, J. Adaptive Hybrid Maximum Power Point Tracking Method for a Photovoltaic System. IEEE Trans. Energy Convers. 2013, 28, 353-360. [CrossRef]

15. Ahmed, J.; Salam, Z. An Enhanced Adaptive P\&O MPPT for Fast and Efficient Tracking under Varying Environmental Conditions IEEE Trans. Sustain. Energy 2018, 9, 1487-1496. [CrossRef]

16. Ahmed, J.; Salam, Z. A Modified P\&O Maximum Power Point Tracking Method with Reduced Steady-State Oscillation and Improved Tracking Efficiency. IEEE Trans. Sustain. Energy 2016, 7, 1506-1515. [CrossRef]

17. Sher, H.A.; Murtaza, A.F.; Noman, A.; Addoweesh, K.E.; Al-Haddad, K.; Chiaberge, M. A New Sensorless Hybrid MPPT Algorithm Based on Fractional Short-Circuit Current Measurement and P\&O MPPT. IEEE Trans. Sustain. Energy 2015, 6, 1426-1434. [CrossRef]

18. Killi, M.; Samanta, S. Modified Perturb and Observe MPPT Algorithm for Drift Avoidance in Photovoltaic Systems. IEEE Trans. Ind. Electron. 2015, 62, 5549-5559. [CrossRef]

19. Escobar, G.; Pettersson, S.; Ho, C.N.M.; Rico-Camacho, R. Multisampling Maximum Power Point Tracker (MS-MPPT) to Compensate Irradiance and Temperature Changes. IEEE Trans. Sustain. Energy 2017, 8, 1096-1105. [CrossRef]

20. Sera, D.; Kerekes, T.; Teodorescu, R.; Blaabjerg, F. Improved MPPT method for rapidly changing environmental conditions. In Proceedings of the 2006 IEEE International Symposium on Industrial Electronics, Montreal, QC, Canada, 9-13 July 2006; Volume 2, pp. 1420-1425. [CrossRef]

21. Sera, D.; Teodorescu, R.; Hantschel, J.; Knoll, M. Optimized Maximum Power Point Tracker for fast changing environmental conditions. In Proceedings of the 2008 IEEE International Symposium on Industrial Electronics, Cambridge, UK, 30 June-2 July 2008 ; pp. 2401-2407. [CrossRef]

22. Bazzi, A.M.; Krein, P.T. Concerning "Maximum Power Point Tracking for Photovoltaic Optimization Using Ripple-Based Extremum Seeking Control". IEEE Trans. Power Electron. 2011, 26, 1611-1612. [CrossRef]

23. Zurbriggen, I.G.; Ordonez, M. PV Energy Harvesting Under Extremely Fast Changing Irradiance: State-Plane Direct MPPT. IEEE Trans. Ind. Electron. 2018, 66, 1852-1861. [CrossRef]

24. Casadei, D.; Grandi, G.; Rossi, C. Single-Phase Single-Stage Photovoltaic Generation System Based on a Ripple Correlation Control Maximum Power Point Tracking. IEEE Trans. Energy Convers. 2006, 21, 562-568. [CrossRef]

25. Hua, C.-C.; Zhan, Y.-J. A Hybrid Maximum Power Point Tracking Method without Oscillations in Steady-State for Photovoltaic Energy Systems. Energies 2021, 14, 5590. [CrossRef]

26. IEA. Will Pumped Storage Hydropower Expand More Quickly than Stationary Battery Storage? Analysis. 2019. Available online: https:/ / www.iea.org/articles/will-pumped-storage-hydropower-expand-more-quickly-than-stationary-battery-storage (accessed on 17 January 2021).

27. Abdulkadir, M.; Yatim, A.H. Hybrid maximum power point tracking technique based on PSO and incremental conductance. In Proceedings of the 2014 IEEE Conference on Energy Conversion (CENCON), Johor Bahru, Malaysia, 13-14 October 2014; pp. 271-276. [CrossRef]

28. Mohanty, S.; Subudhi, B.; Ray, P.K. A Grey Wolf-Assisted Perturb \& Observe MPPT Algorithm for a PV System. IEEE Trans. Energy Convers. 2017, 32, 340-347. [CrossRef]

29. Ram, J.P.; Pillai, D.S.; Rajasekar, N.; Strachan, S.M. Detection and Identification of Global Maximum Power Point Operation in Solar PV Applications Using a Hybrid ELPSO-P\&O Tracking Technique. IEEE J. Emerg. Sel. Top. Power Electron. 2020, 8, 1361-1374. [CrossRef]

30. European Committee for Electrotechnical Standardization (CENELEC). European Standard EN 50530; Overall Efficiency of Grid Connected Photovoltaic Inverters; European Committee for Electrotechnical Standardization: Brussels, Belgium, 2010. 
31. Kivimaki, J.; Kolesnik, S.; Sitbon, M.; Suntio, T.; Kuperman, A. Revisited Perturbation Frequency Design Guideline for Direct Fixed-Step Maximum Power Point Tracking Algorithms. IEEE Trans. Ind. Electron. 2017, 64, 4601-4609. [CrossRef]

32. Femia, N.; Petrone, G.; Spagnuolo, G.; Vitelli, M. Optimization of Perturb and Observe Maximum Power Point Tracking Method. IEEE Trans. Power Electron. 2005, 20, 963-973. [CrossRef]

33. Viinamäki, J.; Jokipii, J.; Messo, T.; Suntio, T.; Sitbon, M.; Kuperman, A. Comprehensive dynamic analysis of photovoltaic generator interfacing DC-DC boost power stage. IET Renew. Power Gener. 2015, 9, 306-314. [CrossRef]

34. Motahhir, S.; El Hammoumi, A.; El Ghzizal, A. The most used MPPT algorithms: Review and the suitable low-cost embedded board for each algorithm. J. Clean. Prod. 2019, 246, 118983. [CrossRef]

35. Bollipo, R.B.; Mikkili, S.; Bonthagorla, P.K. Hybrid, optimization, intelligent and classical PV MPPT techniques: A Review. CSEE J. Power Energy Syst. 2021, 7, 9-33. [CrossRef]

36. Kakosimos, P.E.; Kladas, A.G.; Manias, S.N. Fast Photovoltaic System Voltage or Current Oriented MPPT Employing a Predictive Digital Current-Controlled Converter. IEEE Trans. Ind. Electron. 2013, 60, 5673-5685. [CrossRef]

37. Tang, L.; Wang, X.; Xu, W.; Mu, C.; Zhao, B. Maximum power point tracking strategy for photovoltaic system based on fuzzy information diffusion under partial shading conditions. Sol. Energy 2021, 220, 523-534. [CrossRef] 